Delft University of Technology

\title{
Time-Varying Risk Measurement for Ship Collision Prevention
}

Huang, Yamin; van Gelder, P. H.A.J.M.

DOI

10.1111/risa. 13293

Publication date

2019

Document Version

Accepted author manuscript

Published in

Risk Analysis

\section{Citation (APA)}

Huang, Y., \& van Gelder, P. H. A. J. M. (2019). Time-Varying Risk Measurement for Ship Collision

Prevention. Risk Analysis, 40(1), 24-42. https://doi.org/10.1111/risa.13293

\section{Important note}

To cite this publication, please use the final published version (if applicable).

Please check the document version above.

\section{Copyright}

Other than for strictly personal use, it is not permitted to download, forward or distribute the text or part of it, without the consent of the author(s) and/or copyright holder(s), unless the work is under an open content license such as Creative Commons.

\section{Takedown policy}

Please contact us and provide details if you believe this document breaches copyrights.

We will remove access to the work immediately and investigate your claim. 


\title{
Time-varying Risk Measurement for Ship Collision
}

\section{Prevention}

Yamin Huang*, P.H.A.J.M. van Gelder

\begin{abstract}
We proposed an innovative Time-varying Collision Risk (TCR) measurement for ship collision prevention in this paper. The proposed measurement considers the level of danger of the approaching ships and the capability of a ship to prevent collisions. We define the TCR as the probability of the overlap of ships' positions in the future, given the uncertainty of maneuvers. Two sets are identified: 1) the velocity obstacle set as the maneuvers of the own ship that lead to collisions with target ships, and 2) the reachable velocity set as the maneuvers which the own ship can reach regarding its maneuverability. We then measure the TCR as the time-dependent percentage of overlap between these two sets. Several scenarios are presented to illustrate how the proposed measurement identifies the time-varying risk levels, and how the approach can be used as an intuitively understandable tool for collision avoidance.
\end{abstract}

Keywords: Time-varying collision risk; maneuverability; velocity obstacle; reachability analysis.

Faculty of Technology, Policy and Management, Delft University of Technology, Delft, the Netherlands.

* Address correspondence to Yamin Huang, Faculty of Technology, Policy and Management, Delft University of Technology, Jaffalaan 5, 2628 BX Delft, the Netherlands; tel: +31 1527 85143; y.huang-3@tudelft.nl or y.m.huang@outlook.com 


\section{INTRODUCTION}

Ship collisions frequently occur at sea, which often results in severe consequences, such as loss of life, damage to property and environmental pollution. Reports and literature(Chauvin, Lardjane, Morel, Clostermann, \& Langard, 2013; Grech, Horberry, \& Smith, 2002; Martins \& Maturana, 2010) have unveiled that human error is one of the major causes of collisions, especially the lack of situational awareness. Thus, one proactive action to prevent this type of accident is enhancing the situational awareness of the Officer On Watch (OOW), in particular, supporting them to detect threats and find a collision-free solution as early as possible (Goerlandt, Montewka, Kuzmin, \& Kujala, 2015). As the first step, a time-varying risk measurement is fundamental to perform this task.

Ship collision risk measurements are usually from the perspective of one ship, denoted as Own Ship (OS), and focus on evaluating the levels of danger of approaching ships (Goerlandt et al., 2015; LopezSantander \& Lawry, 2016; Tam \& Bucknall, 2010). In existing measurements, the maneuver feature of the OS is neglected. However, a good maneuverability contributes to the safety of a ship(Y. Li, Landsburg, Barr, \& Cahsal, 2005). Better maneuverability means the OOWs have more maneuver options than the one with poor maneuverability. More options mean that the ship has a higher chance to avoid a collision and, therefore, a lower risk of collision. The maneuvers, then, can be regarded as a "barrier" to prevent the "top event" (i.e., collisions) from the "threats" (i.e. the approaching ships). From this viewpoint, the traditional approaches only measure the danger levels of the "threats" and ignore the capability of the OS to reduce the "threats". In return, the relevant collision risk measurements cannot help the OOWs identify the real danger that is difficult to avoid.

In this article, we propose a risk measurement which considers both "threats" and "barriers", i.e., the danger level of the approaching ships and the OS's maneuverability. Collision risk is defined as the probability of collisions, given an uncertainty in the OS's maneuvers. The risk is formulated as the percentage of the achievable maneuvers which leading to collisions. The maneuver refers to course and speed of the OS, in this article. This risk measurement reflects the OS's room for maneuver to avoid 
collisions: a higher collision risk indicates fewer feasible maneuvers to avoid a collision, and more confident that the collision will happen; a lower risk means more collision-free maneuvers, which implies that more chances to avoid the collision.

This measurement can help the OOWs to identify which danger is urgent, and help them be aware of the room for maneuver. Moreover, collecting and extrapolating the risk in time show the development of the risk, e.g., increasing or decreasing. Those could help the OOWs make decisions.

The remainder of this paper is organized as follows: Section 2 provides an overview of the existing collision risk measurements. In Section 3, we propose a time-varying collision risk measurement. The construction of reachable set is illustrated in Section 4. We simulate different scenarios in Section 5 to show the potential of the proposed measurement. The discussions on uncertainties are presented in Section 6. In the end, in Section 7, the conclusions and the future studies are given.

\section{LITERATURE REVIEW}

Ship collision has received numerous attention, due to its high frequency and huge potential losses. Various measurements have been proposed to assess the collision risk at sea. Two different perspectives are found in the literature. One measures the risk from the perspective of waterway management, which concerns the probability and consequence of collision events in specific waters(Montewka et al., 2014; J. Zhang, Teixeira, Guedes Soares, Yan, \& Liu, 2016) (Readers who are interested in this topic can find more details in the literature (S. Li, Meng, \& Qu, 2012) and (Goerlandt \& Montewka, 2015)). In this paper, we focus on the other one, which evaluates the collision risk from the perspective of the operators, e.g. the OOWs. These measurements usually assess the probability of collision between the OS and the target ships (TSs) and the measured risk usually is devoted to collision prevention for one ship(Goerlandt et al., 2015; Lopez-Santander \& Lawry, 2016). The applications of these measurements are involved in collision alert system(Goerlandt et al., 2015) and path planning(Y. Liu, Liu, Song, \& Bucknall, 2017). Moreover, the measurements can not only improve the situational awareness for the OOWs, but also support the unmanned ship to prevent collision(Lambert, Gruyer, \& Saint Pierre, 2008; Y. Liu et al., 2017). 
In existing risk-related studies, two different cultures are often mentioned, namely subjective risk and objective risk(Hermansson, 2012). The two cultures have been brought to real-time collision risk measurement.

In some research, collision risk is defined as subjective risk and measured based on the perception of the experts, such as the captains (Lopez-Santander \& Lawry, 2016), pilots (Chin \& Debnath, 2009), etc. The aim of these measurements is training a risk measurement to approach the experts' feeling of dangers in different scenarios. Specifically, this approach is to summarize the observed data into the values of several variables, and then it uses expert judgment based on the experience (Ahn, Rhee, \& You, 2012; LopezSantander \& Lawry, 2016) and navigation rules (Goerlandt et al., 2015; Lopez-Santander \& Lawry, 2016) to combine these value into a single assessment of the collision risk. The collision alarm is then triggered by comparison of the risk level with a certain threshold that also relies on expert judgments (Baldauf, Benedict, Fischer, Motz, \& Schröder-Hinrichs, 2011; Hilgert \& Baldauf, 1997). In a word, the measured collision risk represents the degree of experts' belief on the occurrence of the collisions. The variables that have been used including: motion related variables, such as relative position (Szlapczynski \& Szlapczynska, 2016), relative speed (W. Zhang, Goerlandt, Montewka, \& Kujala, 2015), relative bearing (Tam \& Bucknall, 2010), types of encountering (Goerlandt et al., 2015; Lopez-Santander \& Lawry, 2016), Distance at Closet Point of Approach (DCPA) and Time to Closet Point of Approach (TCPA)(Chin \& Debnath, 2009), ship domain (Szlapczynski \& Szlapczynska, 2016; Wang, 2010), etc.; descriptions of the TSs, such as ship length (Wang, 2010), ship type (Goerlandt et al., 2015), gross tonnage (Chin \& Debnath, 2009), etc.; states of environment, such as wave conditions (Kao, Lee, Chang, \& Ko, 2006), visibility (Goerlandt et al., 2015), etc. For further reading, the readers can find literature (Tam, Bucknall, \& Greig, 2009).

Other researchers regard collision risk as objective risk and employ probabilistic approaches to measure the risk. A collision is seen as a physical process that ships approach each other and their positions overlap at the end (Belkhouche \& Bendjilali, 2012; Park \& Kim, 2016; Simsir, Amasyali, Bal, Çelebi, \& Ertugrul, 2014). The risk is the probability of overlap in the future, given initial states, ship models and uncertainties (Belkhouche \& Bendjilali, 2012). Given ship motion model and initial states, trajectories of ships are 
deterministic and collision event is certain. However, the uncertainties of the system are inevitable, e.g. uncertainties in modeling models (mainly environmental disturbance (Belkhouche \& Bendjilali, 2012)), sensing data (Park \& Kim, 2016), etc. Consequently, the trajectories of ships become probabilistic. Provided the probabilistic trajectories, the probability of overlap can be estimated, i.e. the collision risk. From this point of view, the probability of collision is determined by the introduced uncertainties. Thus, the risk measurement is to assess the effect of the uncertainties in the physical process. In brief, the measured collision risk is a conditional probability of collision given uncertainties.

In these studies, the measurements focus on assessing the safety level of the approaching threats (i.e., TSs), while the capability of the OS to prevent a collision has been ignored, i.e. the maneuverability. As a result, the measured probability of collision cannot reflect the real danger. For example, a ship with good maneuverability might be able to avoid a collision with a ship close by, while a ship with poor maneuverability might not in the same scenario. Therefore, in this paper, we assess the probability of collision considering the maneuverability of the OS. The risk is expected to facilitate the OOWs to find the real collision dangers which are difficult to avoid.

\section{TIME-VARYING COLLISION RISK MEASUREMENT}

\subsection{Definition of the Time-varying Collision Risk}

There is no agreement on risk definition (Aven, 2012), but the risk is usually tied to probability (or uncertainty) and consequences. Researchers are more interested in the probability of an unwanted event for the purpose of preventing accidents (Goerlandt et al., 2015). In this work, therefore, we accept the definition of risk based on probability concepts(Andretta, 2014).

\section{Definition (1): Risk is the probability of an unwanted event.}

The unwanted event refers to a ship-collision event in this article. In each collision event, at least two ships are involved, i.e., an OS and a TS. Since the states of ships are time-varying, the probability of collision is time-varying as well. Thus, we have time-varying collision risk (TCR). 


\section{Definition (2): TCR is the time-dependent probability of the event that OS cannot avoid a} collision with TSs.

\subsection{The Framework of TCR Measurement}

In TCR measurement, the OS is modeled as a dynamic system. The position of the OS, say $x$, is updated by its velocity $\vec{v}$, i.e. $\dot{x}=\vec{v}$. A collision is the overlap of the position of the OS and a TS. Let $\chi$ be a set of the OS's states that triggers collisions, i.e. the un-safe set. Then, a collision is described as an event that the trajectory of the system (the solid curve in Fig. 1) crosses an un-safe set $\chi$ (the red region in Fig. 1), i.e. $\exists t \geq t_{0}, x(t) \in \chi$.

With different maneuvers, the trajectory of the system might cross or avoid the un-safe set, e.g., the dashed lines in Fig. 1. Hence, given an uncertainty in the OS's maneuvers, TCR at time $t$ can be formulated as a probability of maneuvers leading the trajectory to cross the un-safe sets, i.e.,

$$
\operatorname{TCR}(t)=\sum_{i=1}^{n} P\left(\text { collision } \mid \vec{v}_{i}\right) \cdot P\left(\vec{v}_{i}\right)
$$

where $P\left(\vec{v}_{i}\right)$ is the probability of choosing velocity $\vec{v}_{i}$;

$P\left(\right.$ collision $\left.\mid \vec{v}_{i}\right)$ is the probability of collision given $\vec{v}_{i}$;

$n$ is the total number of reachable velocities.

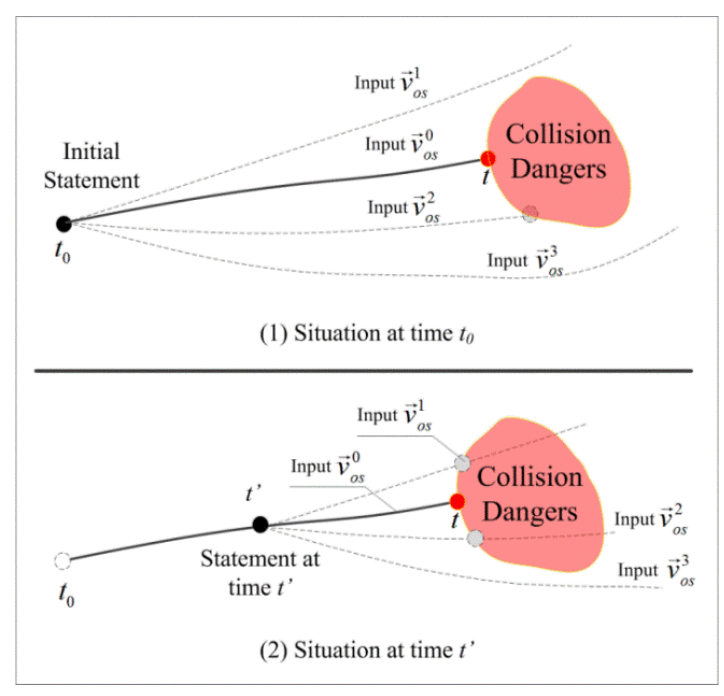

Fig. 1. The illustration of a collision process in OS's state space 
Fig. 1 illustrates the idea of TCR measurement. A collision will happen at time $t$, if no evasive actions are taken. Any maneuvers taken prior to time $t$ will change the trajectories of OS. Different maneuvers could lead to different results, e.g.: pass safely, near miss, serious damage. Assume that the un-safe set (the red region in Fig. 1) is known and stationary; the ship in Fig.1 has 4 feasible maneuvers at each time, and each maneuver has an equal chance. In Fig. 1(1), at time $t_{0}$, the system has two available solutions which can help the OS to avoid the collision. Thus, the TCR at time $t_{0}$ is 0.5 . However, as time passes, the number of collision-free solutions decreases. In Fig. 1(2) at time $t^{\prime}$, only one maneuver is available for collision avoidance, say $\vec{v}_{O S}^{3}$. Therefore, the TCR at time $t^{\prime}$ increases to 0.75 .

\subsection{Implementation of the TCR Measurement}

For the sake of convenient calculation, in this article, we make two assumptions to simplify the TCR measurement in Equation (1):

Assumption (1): The probability of choosing maneuvers yield to a uniform distribution.

Assumption (2): The trajectories of the TSs are known and linear (i.e., the TSs only follow their initial velocities).

From Assumption (1), $P\left(\vec{v}_{i}\right)$ in Equation (1) is assumed to meet a uniform distribution, which means the probabilities of choosing each maneuver are equal. In fact, the distribution of maneuvers in calm water depends on two factors: the OOW's preference and the actuator errors. These two factors are neglected in this paper. The OOW's preference differs greatly from one to another, and actuator errors need numerous observation data. The lack of data makes it difficult to fit a proper distribution by far. Therefore, a uniform distribution is a reasonable choice, as the principle of maximum entropy. Other distributions can be applied as well, when further information is available, e.g. OOW's preference and actuator errors.

From Assumption (2), the motion of the TS is assumed to be known, which makes the un-safe sets certain and invariant. Subsequently, given a $\vec{v}_{i}$, there is only one result: the trajectory of the OS crosses the un-safe set, then $P\left(\right.$ collision $\left.\mid \vec{v}_{i}\right)=1$, or the trajectory avoids the un-safe set, which $P\left(\right.$ collision $\left.\mid \vec{v}_{i}\right)=0$. 
Providing these settings, the probability of collision for the OS can be depicted as the proportion of maneuvers leading to collisions to its all feasible maneuvers before a collision:

$$
\operatorname{TCR}(t)=\sum_{i=1}^{n} P\left(\text { collision } \mid \vec{v}_{i}\right) \cdot P\left(\vec{v}_{i}\right)=\frac{n_{\text {collision }}(t)}{n(t)}
$$

where $n_{\text {collision }}(t)$ is the number of velocities leading to collisions at time $\mathrm{t} ; n(t)$ is the number of reachable velocities before collisions at time $t$.

There are two keys to calculate the TCR in Equation (2): 1) identify all the velocities lead to collisions; 2) find all the reachable velocities before the collision. Thus, we define two sets:

Definition (3): Velocity Obstacle set (VO set) is a set of velocities of the OS which could lead to collisions between the OS and the TSs.

Definition (4): Reachable Velocity set (RV set) is a set of velocities that OS can reach before the collisions.

Both sets can be shown in the OS's velocity space. In Fig. 2, the velocity space is described in the form of speed and course. The VO set is depicted as a red cone. The RV is represented as a green zone. The RV set is divided into several sub-areas by VO set, namely $S_{r}, S_{c}$, and $S_{l}$. The sub-area $S_{c}$ is the intersection of VO set and RV set. It is a set of velocities that the OS can reach, but could lead to collision:

$$
S_{c}=\left\{\vec{v}_{i} \mid\left(\vec{v}_{i} \in \mathrm{RV}\right) \cap\left(\vec{v}_{i} \in \mathrm{VO}\right)\right\} .
$$

The remaining sub-areas belong to RV set are collectively noted as $\bar{S}_{c}$. The velocity in this set is reachable for the OS, and it helps the OS to avoid the collision:

$$
\bar{S}_{c}=\left\{\vec{v}_{i} \mid\left(\vec{v}_{i} \in \mathrm{RV}\right) \cap\left(\vec{v}_{i} \notin \mathrm{VO}\right)\right\} .
$$

According to Equation (2), the TCR for the OS can be interpreted as the proportion of the overlap $\left(S_{c}\right)$ to the RV set $\left(S_{c}+\bar{S}_{c}\right)$ :

$$
\operatorname{TCR}(t)=\frac{\mathrm{N}\left(S_{c}(t)\right)}{\mathrm{N}\left(S_{c}(t)+\bar{S}_{c}(t)\right)},
$$


where $\mathrm{N}(\cdot)$ means the size of a set; $S_{c}(t)$ and $\bar{S}_{c}(t)$ refer to corresponding sub-areas at time $t$.

A bigger sub-set $S_{c}(t)$ on RV set shows the fewer solutions for OS avoiding the collision, which indicates a higher risk $(T C R(t))$; a smaller $S_{c}(t)$ implies adequate collision-free solutions and means a lower risk $(T C R(t))$.

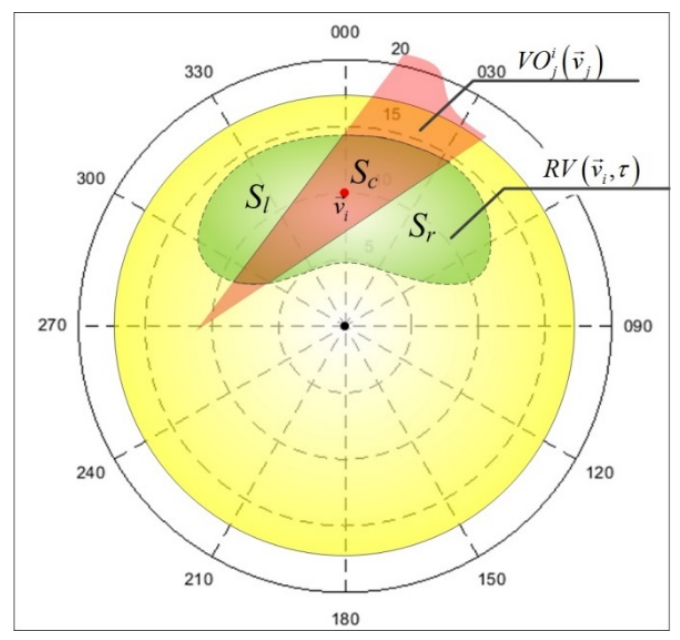

Fig. 2. The VO set and the RV set in velocity space of the OS (note: a point in this space represents a velocity of the OS: the axes indicate the course; the distance from the pole represents the speed)

\subsubsection{VO set in Velocity Space}

The VO set is obtained by the Velocity Obstacle algorithm (VO algorithm). The VO algorithm is one of the popular methods to identify the conflict velocity for the vehicle(van den Berg, Lin, \& Manocha, 2008). It has been applied in many fields, such as robot motion planning(Fiorini \& Shiller, 1998), aviation collision avoidance(Velasco, Borst, Ellerbroek, van Paassen, \& Mulder, 2015), etc. The core of the VO algorithm is mapping a moving obstacle from the geographical space to the velocity space. In this way, the moving obstacle turns to be a static cone in the velocity space which contains all the velocities leading to collisions. For details of the VO algorithm, we refer to literature (Fiorini \& Shiller, 1998). Details of using the VO algorithm in the maritime domain can be found in the literature (Huang, Chen, \& van Gelder, 2019), (Huang \& Gelder, 2017) and (Kuwata, Wolf, Zarzhitsky, \& Huntsberger, 2014).

Applying the VO algorithm to the TCR measurement, the following assumption is made: 


\section{Assumption (3): The shape of ships is represented as circles in which the length of the ship is its diameter;}

The Assumption (3) is introduced to make the collision cone independent from ships' shape, and the headings of ships would not impact on the shape of VO set.

\subsection{2. $R V$ Set in Velocity Space}

All the velocities out of the VO set are collision-free, according to VO algorithm. However, not all these velocities are reachable regarding the time and the maneuvering ability of the OS. Therefore, a RV set is necessary to figure out all the reachable velocities of the OS.

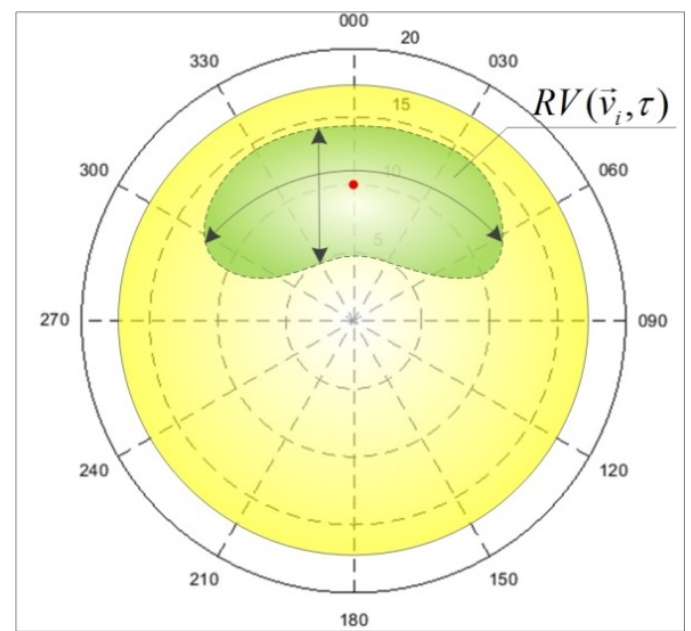

Fig. 3. The RV set in velocity space of the OS

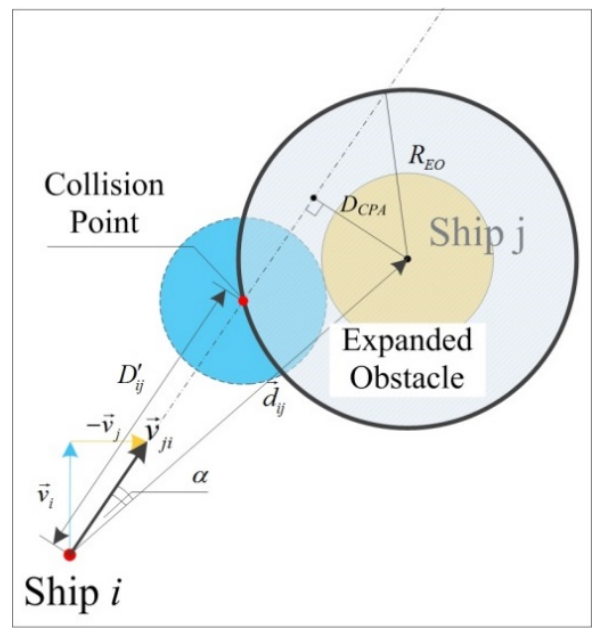

Fig. 4. TTC calculation.

A RV set with a time window $\tau$ and an initial velocity $\vec{v}_{i}$ is noted as $R V\left(\vec{v}_{i}, \tau\right)$, see Fig. 3. The yellow zone is the RV set of the OS regardless of the TTC (Time To Collison). The green zone is the RV set considering the TTC (i.e., $R V\left(\vec{v}_{i}, \tau=\mathrm{TTC}\right)$ ). The angular range of $R V\left(\vec{v}_{i}, \tau\right)$ reflects the turning ability of the OS, and the radius range of $R V\left(\vec{v}_{i}, \tau\right)$ shows the acceleration/deceleration ability. A bigger time window or a better maneuverability contributes to a larger RV set.

The construction of RV set is, in fact, the reachability analysis of a dynamic system (i.e., OS) considering the TTC and the ship maneuverability. The calculation of TTC and the basic concept of ship 
maneuvering ability are introduced in this section. The details of reachability analysis for constructing a RV set is shown in Section 4.

TTC is the time left to avoid a collision. The calculation of the TTC is explained in Fig. 4 related to the scenario in which two ships encounter. Setting the TS as the reference, expanding the TS's scale by the OS's dimension (noted as Expanded Obstacle (EO)), the collision scenario turns out to be a particle which keeps moving with a relative velocity and colliding with a static EO at "Collision Point". Thus, the TTC can be formulated as:

$$
T T C=\frac{D_{i j}^{\prime}}{\left\|\vec{v}_{j i}\right\|}
$$

where $D_{i j}^{\prime}=\left\|\vec{d}_{i j}\right\| \cos (\alpha)-\sqrt{R_{E O}{ }^{2}-D_{C P A}{ }^{2}}$ with $\vec{d}_{i j}=P_{j}-P_{i}, \cos (\alpha)=\frac{\vec{d}_{i j} \cdot \vec{v}_{j i}}{\left\|\vec{d}_{i j}\right\|\left\|\vec{v}_{j i}\right\|}$, and $D_{C P A}=\left\|\vec{d}_{i j}\right\| \sqrt{1-\cos ^{2}(\alpha)}$.

Ship maneuvering ability is a significant factor that influences the size of a RV set. Generally, ship maneuvering ability refers to the turning ability, course-keeping ability, stopping ability, etc.(J. Liu, Hekkenberg, Rotteveel, \& Hopman, 2015). In this article, we focus on the changes in ship's course (turning ability) and speed (acceleration/deceleration ability). Moreover, we hold two assumptions on ship maneuvering ability:

Assumption (4): The speed-loss effect during the turning phase is neglected, i.e. the ship is capable to keep its speed during turning;

Assumption (5): The external disturbance from wind, current, etc. are excluded in this paper. Therefore, the ship's position can be predicted with its velocity only.

These assumptions aim at simplifying the ship motion model. The assumption (4) allows us to consider the acceleration process and turning process separately. The assumption (5) is made to exclude the impacts of environmental disturbances on ship's motion. 


\section{REACHABLE VELOCITY SET CONSTRUCTION}

The construction of the RV set is based on reachability analysis. Reachability analysis is a method to find a set of states which the system can reach under constraints. The reachability analysis has been applied in many fields: planning paths for robots (Vendittelli, Laumond, \& Nissoux, 1999); detecting dangers for aircrafts(Sahawneh \& Beard, 2014), verifying safety for autonomous cars (Althof, 2010), etc.

\subsection{Problem Statement}

The main problem of the RV set construction is: find all velocities which the OS can achieve, given an initial velocity $\left(\vec{v}_{0}\right)$ and time window $(\tau)$.
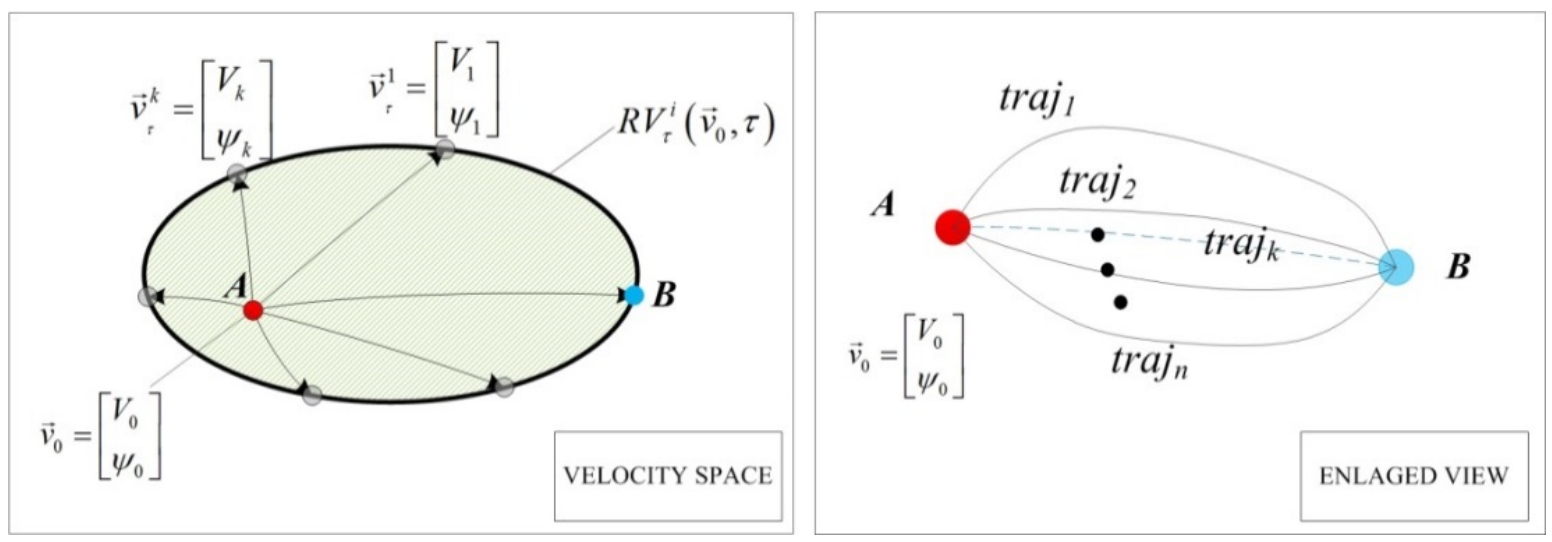

Fig. 5 The illustration of a reachable velocity set of OS (notes: the vectors in the space are the changes in velocity; $\vec{v}_{k}$ is a velocity $k$, in the form of speed $V_{k}$ and course $\psi_{k}$ )

An illustration of the RV set is shown in Fig. 5. The green ellipse is a RV set of OS, with respect to an initial velocity $\left(\vec{v}_{0}\right)$ and a certain time window $\tau$. All the velocities in this set are reachable for the OS, while the rest are not reachable in time $\tau$. The point "A" represents the initial velocity, and the point "B" is an arbitrary velocity. The OS wants to modify its velocity from "A" to "B". Numerous controls can achieve this goal and we denote them as $U^{A \rightarrow B} \cdot u_{i} \in U^{A \rightarrow B}$ is a control input which leads OS's velocity move from "A" to "B"; $\operatorname{traj}_{i}$ is the path how the velocity changes. $t_{\text {traj }}$ is the time needed for the velocity 
move from "A" to "B" along $\operatorname{traj}_{i}$. Within these controls, we can find an optimal one (say $u_{k}^{\text {opt }}$ ) which minimizes the time:

$$
t_{k}^{o p t} \square \min \left\{t_{\text {traj }}\left(u_{i}^{o p t}\right) \mid u_{i}^{o p t} \in U^{A \rightarrow B}\right\} .
$$

If $\tau \geq t_{k}^{\text {opt }}$, at least one control can complete this mission within $\tau$, and " $\mathrm{B}$ " is contained in $R V\left(\vec{v}_{0}, \tau\right)$; if $\tau<t_{k}^{\text {opt }}$, no controls can change the velocity from "A" to "B" within $\tau$, which means that "B" is out of $R V\left(\vec{v}_{0}, \tau\right)$; if $\tau=t_{k}^{\text {opt }}$, point "B" is located on the boundary of the RV set $R V\left(\vec{v}_{0}, \tau=t_{k}^{\text {opt }}\right)$.

Therefore, the main problem of RV set construction is equivalent to finding a minimum operation time from an initial velocity to all other velocities. Therefore, $R V\left(\vec{v}_{0}, \tau\right)$ is a collection of the velocity whose minimum operation time smaller than $\tau$.

\subsection{Steps of RV Set Construction}

To construct a RV set, firstly, we need to find the minimum operational time $t^{\text {opt }}$ for each velocity in the velocity space. This velocity is on the boundary of the set $R V\left(\vec{v}_{0}, \tau=t_{k}^{\text {opt }}\right)$, which is also contained in the set $R V\left(\vec{v}_{0}, \tau \geq t_{k}^{\text {opt }}\right)$. The $R V\left(\vec{v}_{0}, \tau\right)$ is a collection of velocities whose $t_{k}^{\text {opt }}<\tau$. Therefore, the steps of RV set construction are as follows:

Step (1) Gridding: Grid the velocity space of OS, say: $\vec{v}_{\text {alter. }}: \square^{m} \times \square^{n}$ and $\vec{v}_{\text {alter. }} \square\left[\begin{array}{l}v \in\left[0, v_{\max }\right] \\ \psi \in[-\pi, \pi]\end{array}\right]$;

Step (2) Initialization: Initialize the OS's current velocity, say $\vec{v}_{0} \square\left[\begin{array}{l}V_{0} \\ \psi_{0}\end{array}\right]=\left[\begin{array}{l}V_{0} \\ 0\end{array}\right]$;

Step (3) Target velocity setting: Choose one vector in velocity space ( $\left.\vec{v}_{\text {alter. }}\right)$ and set it as $\vec{v}_{\text {target }}$;

Step (4) Strategy searching: Find an optimal control input $u_{k}^{o p t}$;

Step (5) Calculation: Calculate the time for the control $t_{k}^{\text {opt }}$, and store it in a m-by-n matrix $T_{\text {target }}$; 
Step (6) Loop: If the $\vec{v}_{\text {target }}$ is not the last element in $\vec{v}_{\text {alter. }}$, go back to Step (3); otherwise, quit the loop.

Step (7) RV set determination: The RV set for any time window $\tau$ is a set of velocities whose operational time $t_{k}^{\text {opt }}$ is not larger than $\tau$.

In this method, only the operational time for the optimal control is needed; the RV sets for any time window $\tau$ are calculated at the same time. Given an initial velocity, it is unnecessary to recalculate the RV set for a different time window $\tau$.

\subsubsection{Ship Motion Model}

The calculation of operational time $t_{t r a j_{i}}$ is strongly tied to the ship's motion model. Ship motion is related to acceleration, velocity, and yaw rate. According to literature (Crane, Eda, \& Landsburg, 1989), when the initial speed of the ship is larger than 0 , the speed and the acceleration have a relation similar to the quadratic relation. Moreover, the yaw rate has a positive and linear relationship with speed. Therefore, we can formulate our ship motion model by acceleration equation (Equation (8)) and turning equation (Equation (9)). These dynamic equations describe a simplified ship motion model in which turning process and acceleration process are decoupled (Assumption (4)). Moreover, the ship can obtain a constant yaw rate when it decides to turn, which means this ship can turn in a circle whose radius is $v(t) / r(t)$.

$$
\begin{aligned}
& a=\dot{v}=p_{1} v^{2}+p_{2} v+p_{3}, \\
& r=\dot{\psi}=k \cdot v,
\end{aligned}
$$

where $a$ is the acceleration $\left(\left[\mathrm{m} / \mathrm{s}^{2}\right]\right)$;

$v$ is speed of the OS $([\mathrm{m} / \mathrm{s}])$;

$p_{1}, p_{2}$, and $p_{3}$ are the ship coefficients;

$r$ is the yaw rate $([\% / \mathrm{s}])$;

$\psi$ is the course of the $\mathrm{OS}\left(\left[^{\circ}\right]\right)$ 
$k$ is the turning coefficient.

\subsubsection{Control strategies}

To reach the desired velocity, different control strategies should be applied. In this paper, we choose three control strategies:

Strategy 1. Changing the speed first and then turning the course;

Strategy 2. Turning first and then changing the speed;

Strategy $3 . \quad$ Changing the speed and turning at the same time.

Fig. 6 shows how these strategies can steer the velocity towards a target velocity in the velocity space. In this article, the strategy whose operation time is minimum is the optimal one. Denote $T_{1}, T_{2}$, and $T_{3}$ as the time for applying Strategy 1,2 and 3. The calculation of $T_{1}, T_{2}$, and $T_{3}$ is explained in the following parts.
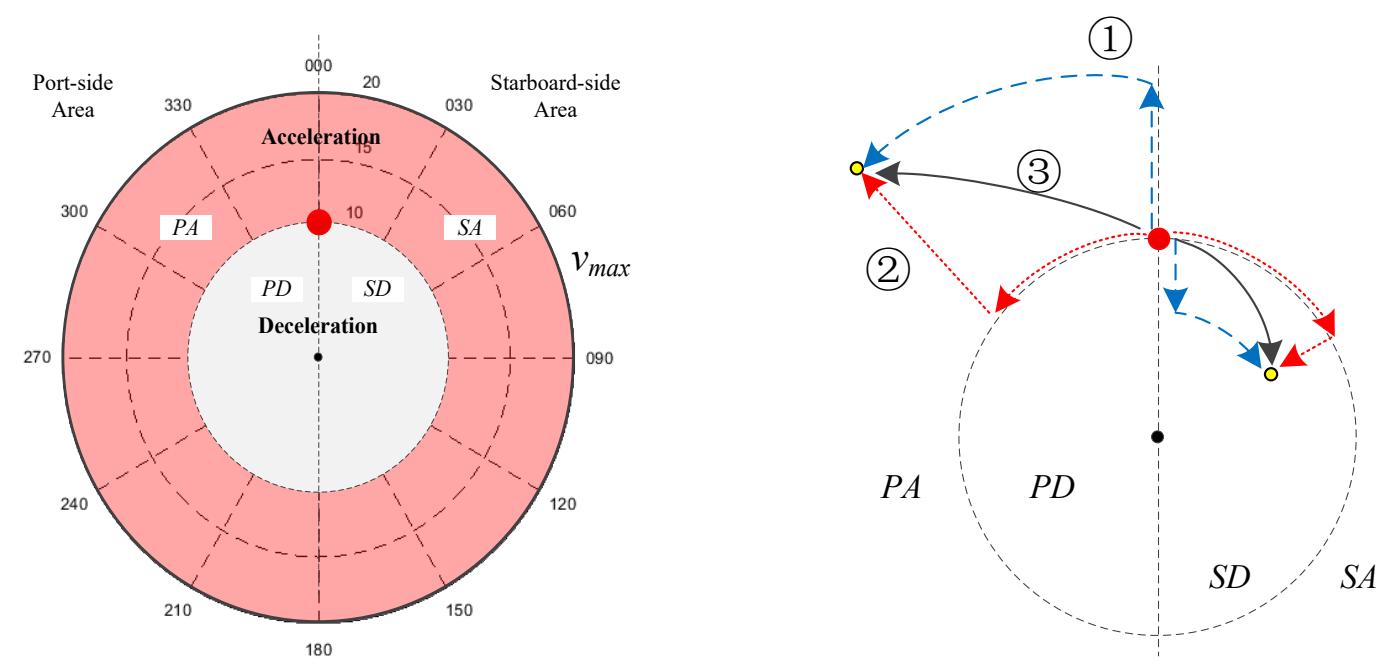

Fig. 6. The illustration of different steering strategies in velocity space (notes: the red point is initial velocity: speed $=10$ knots, course $=0 \mathrm{deg}$ )

Given an initial velocity of the OS, the velocity space is divided into four parts (as shown in Fig. 6):

- Port-side Acceleration (PA): $\vec{v}_{\text {alter }}$ in this part needs port-side turning and acceleration;

- Port-side Deceleration (PD): $\vec{v}_{\text {alter. }}$ in this part needs port-side turning and deceleration;

- Starboard-side Acceleration (SA): $\vec{v}_{\text {alter. }}$ in this part needs starboard-side turning and acceleration; 
- Starboard-side Deceleration (SD): $\vec{v}_{\text {alter. }}$ in this part needs starboard-side turning and deceleration.

According to this deviation, two major situations can be identified. Giving an initial velocity is $\vec{v}_{0}=\left[V_{0}, \psi_{0}\right]^{\prime}$ and a target velocity is $\vec{v}_{\text {target }}=\left[V_{1}, \psi_{1}\right]^{\prime}$,

- Acceleration: $\vec{v}_{\text {target }}=\vec{v}_{\text {alter. }} \in\{\mathrm{PA} \cup \mathrm{SA}\}, V_{l}>V_{0}$;

- Deceleration: $\vec{v}_{\text {target }}=\vec{v}_{\text {alter. }} \in\{P D \cup S D\}, V_{l}<V_{0}$.

For the general acceleration cases, the time for these strategies can be calculated as:

$$
\begin{aligned}
& T_{1}=T_{\text {Accel1 }}+T_{\text {Turn } 1}=T_{\text {Accel }}+\frac{\Delta \psi}{k V_{1}} ; \\
& T_{2}=T_{\text {Turn2 }}+T_{\text {Accel2 }}=\frac{\Delta \psi}{k V_{0}}+T_{\text {Accel }} ; \\
& T_{3}= \begin{cases}T_{\text {Accel }}, & \text { when } T_{\text {Accel }} \geq T_{\text {Turn }} \\
T_{\text {Accel }}+\frac{\Delta \psi-\Delta \psi_{\text {Accel }}}{k V_{1}}, & \text { when } T_{\text {Accel }}<T_{\text {Turn }} .\end{cases}
\end{aligned}
$$

where $\Delta \psi$ is the angular difference between $\psi_{0}$ and $\psi_{1}$;

$\Delta \psi_{\text {Accel }}$ is the turning course during the acceleration;

$T_{\text {Turn }}$ is the time for turning process, and it is related to the angular difference (Equation (11));

$T_{\text {Accel }}$ is the time for accelerating, and it can be calculated with Equation (12).

$$
\begin{aligned}
& T_{\text {Turn }}=\frac{\Delta \psi}{r}=\frac{\Delta \psi}{k \cdot V}, \\
& T_{\text {Accel }}=g\left(V_{0}, V_{1}\right) .
\end{aligned}
$$

According to Equation (11), turning with a lower speed needs a longer turning time. From Equation (10), we observe that the time for acceleration in each strategy is the same. Since, $V_{l}>V_{0}$, we have $T_{\text {Turn1 }}<T_{\text {Turn2 }}$, and therefore, $T_{1}<T_{2}$. In Equation (10), $\Delta \psi-\Delta \psi_{\text {Accel }}$ is the rest of course to the target 
course. Since $\frac{\Delta \psi-\Delta \psi_{\text {Accel }}}{k V_{1}}<\frac{\Delta \psi}{k V_{1}}, T_{3}<T_{1}$. Therefore, we have $T_{3}<T_{1}<T_{2}$. That means, when the target speed $V_{l}$ is larger than the initial speed $V_{0}$, the Strategy 3 is the best strategy.

For the general deceleration cases, the operation time for the three strategies are

$$
\begin{aligned}
& T_{1}=T_{\text {Decel1 }}+T_{\text {Turn1 }}=T_{\text {Decel }}+\frac{\Delta \psi}{k V_{1}} ; \\
& T_{2}=T_{\text {Turn2 }}+T_{\text {Decel2 }}=\frac{\Delta \psi}{k V_{0}}+T_{\text {Decel }} ; \\
& T_{3}= \begin{cases}T_{\text {Decel }} & \text { when } T_{\text {Decel }} \geq T_{\text {Turn }} \\
T_{\text {Decel }}+\frac{\Delta \psi-\Delta \psi_{\text {Decel }},}{k V_{1}}, & \text { when } T_{\text {Decel }}<T_{\text {Turn }} .\end{cases}
\end{aligned}
$$

where $T_{\text {Decel }}$ is the time for decelerating, $T_{\text {Decel }}=h\left(V_{0}, V_{1}\right)$;

$\Delta \psi_{\text {Decel }}$ is the change of course during deceleration.

In the deceleration process, $T_{\text {Decel }}$ is a common factor in each strategy. Since $V_{l}<V_{0}$, we have $T_{\text {Turn } 1}>T_{\text {Turn2 }}$, and therefore, $T_{1}>T_{2}$. In Strategy 3, the ship can reach the target course during deceleration (meaning $T_{3}=T_{\text {Decel }}$ ), Strategy 3 would be the best strategy $\left(T_{3}<T_{2}<T_{1}\right)$. If not, the extra time for steering the course to the desired course should be added and it can be formulated as: $\frac{\Delta \psi-\Delta \psi_{\text {Decel }}}{k V_{1}}$. In this case, $T_{3}<T_{1}$, since $\Delta \psi-\Delta \psi_{\text {Decel }}<\Delta \psi$. Therefore, the operational time in different strategies is sorted as $T_{3}<T_{1}$, $T_{2}<T_{1}$. That means, when the target speed $V_{l}$ is smaller than the initial speed $V_{0}$, the Strategy 1 is not the best strategy.

In summary, to control the OS moving from an initial velocity to an arbitrary target velocity, the optimal strategy is chosen as follows:

- In the acceleration case, applying Strategy 3 always can get the minimum time;

- In the deceleration case, the operation time by the Strategy 2 and Strategy 3 should be calculated, and the smaller one is the optimal one. 
The calculation of $T_{\text {Decel }}, T_{\text {Accel }}$, and $T_{\text {Turn }}$ are introduced underneath.

Calculation of $T_{\text {Decel }}, T_{\text {Accel }}$

The time for acceleration or deceleration can be calculated by solving the ship motion model (Equation (8)) and finding the inverse function for the solution. Given the initial speed $v(0)=v_{0}$, the solution of the differential Equation (8) is:

$$
v(t)=\frac{1}{2 p_{1}}\left\{\sqrt{D} \tan \left(\frac{\sqrt{D}}{2}(t+C)\right)-p_{2}\right\},
$$

where $D=4 p_{1} p_{3}-p_{2}^{2}$

$C$ is a constant, $C=\frac{2}{\sqrt{D}} \operatorname{artan}\left(\frac{2 p_{1} v_{0}+p_{2}}{\sqrt{D}}\right)$.

The inverse function of (14) is the time for acceleration/deceleration:

$$
T_{\text {accel/decel }}=\frac{2}{\sqrt{D}} \operatorname{artan}\left(\frac{2 p_{1} v_{\text {target }}+p_{2}}{\sqrt{D}}\right)-C,
$$

where $v_{\text {target }}=\left\|\vec{v}_{\text {target }}\right\|$ is an alternative speed.

The Equation (15) can be used to calculate the time for acceleration and deceleration process using different groups of the coefficients $\left(p_{1}, p_{2}, p_{3}\right)$ in Equation (15) for calculating acceleration and deceleration are different.

Calculation of $T_{\text {Turn }}$

If the speed (say $v^{*}$ ) does not change during the turning process, e.g., Strategy 1 and 2 , the time for turning is the inverse function of the solution of the differential Equation (9). The solution of Equation (9) is

$$
\psi(t)=\int_{0}^{t} k \cdot v^{*} d t=k \cdot v^{*} \cdot t
$$

Therefore, the time for turning $\left(T_{\text {Turn }}\right)$ is 


$$
T_{\text {Turn }}=\frac{\psi_{\text {target }}-\psi_{0}}{k \cdot v^{*}}=\frac{\psi_{\text {target }}}{k \cdot v^{*}} .
$$

If the speed changes during a turning process, e.g., Strategy 3, we need another equation. Applying Equation (14) into Equation (9) and then we have: $\dot{\psi}(t)=k \cdot v(t)$. Theoretically, solving this equation, we have

$$
\psi(t)=\frac{k}{2 p_{1}}\left\{-2\left[\ln \cos \left(\frac{\sqrt{D}}{2} \cdot(t+C)\right)-\ln \cos \left(\frac{\sqrt{D}}{2} \cdot C\right)\right]-p_{2} t\right\},
$$

where coefficients are dependent on the speed changing process, i.e., acceleration or deceleration.

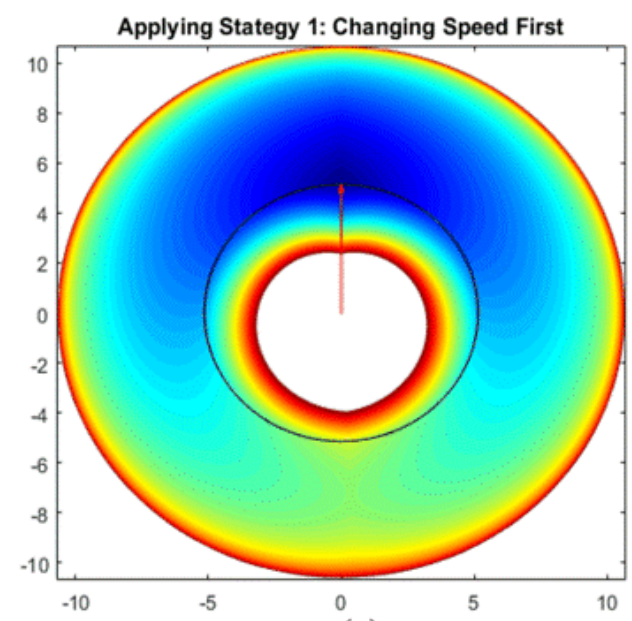

(a)

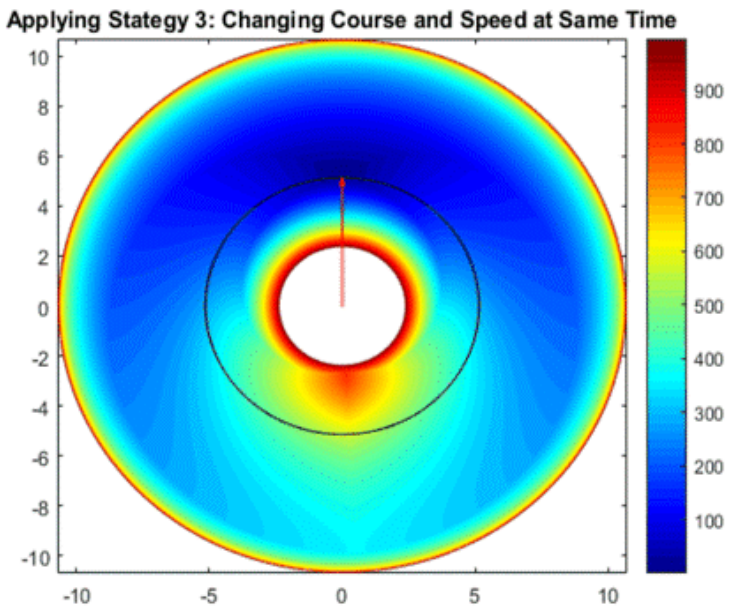

(c)

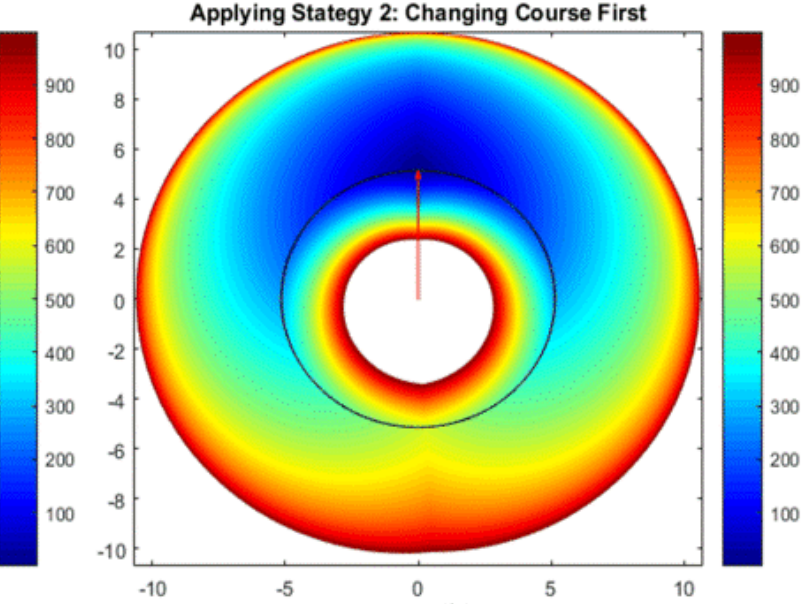

(b)

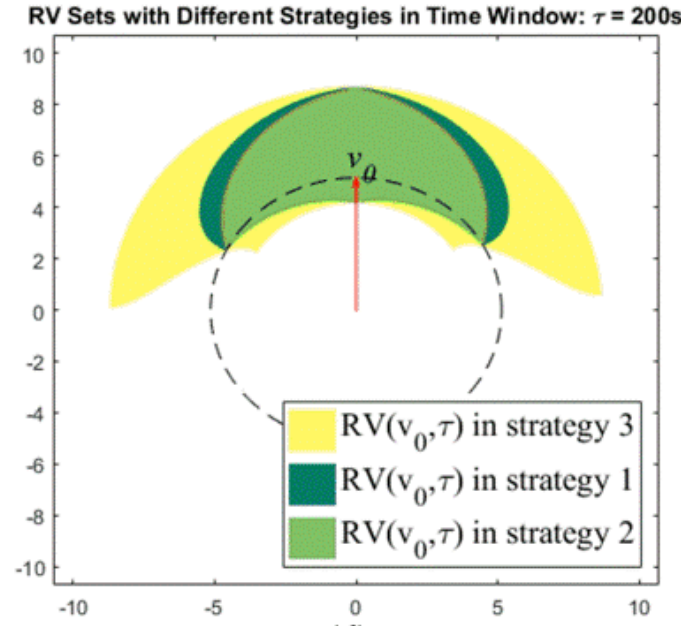

(d)

Fig. 7 The reachable velocity set under different steering strategies (operational time window: 0-1000 seconds; $\left.v_{0}=10[\mathrm{knots}]\right)$ 
To find the inverse function of Equation (18) is complicated. In this paper, a numerical method is applied: firstly, we simulate the turning and acceleration process; secondly, we record the course's response over time under the actions; then we make a regression of the time as a function of course. As a result, for each target course $\psi$, we can find a corresponding operation time for turning and acceleration. The operation time for turning and deceleration is also obtained in this way.

For a better understanding of the constructions of the RV set with different strategies, we provide an example in Fig. 7. Fig. 7 (a) to (c) show the maps of operation time by applying different steering strategies (Strategy 1, 2, 3). Each point in the figure represents a potential target velocity $\left(\vec{v}_{\text {target }}\right)$; the color reflects operation time $\left(t_{k}\right)$ for steering the OS from $\vec{v}_{0}$ to $\vec{v}_{\text {target }}$. With $v_{0}=10$ [knots] and $\tau=200$ [seconds], we can construct three sets of velocities for the three strategies, respectively, as shown in Fig. 7(d). The $R V\left(\vec{v}_{0}, \tau\right)$ with is the union of the three sets, i.e., the set for Strategy 3.

\section{CASE STUDY}

In this section, several scenarios are employed to demonstrate and test the proposed TCR measurement for ship collision prevention. In the simulation, the assumptions in Section 3 are held.

\subsection{Setups}

In the simulation, KVLCC2 is set as the OS. Three types of scenarios are set:

- Basic Scenarios: a series of scenarios involve different encounter situation of two ships;

- Critical Scenarios: a series of scenarios involve the crossing situation of two ships with the same TCPA and different DCPAs;

- Mixed Scenarios: two scenarios involve the encounter of three ships.

- Regulatory Scenario: one scenario that ships comply regulations. 
In these scenarios, the initial speed of the OS is set as $v_{0}=10[\mathrm{knots}] \approx 5.14\left[\mathrm{~m} \cdot \mathrm{s}^{-1}\right]$, and the initial heading is $0 \mathrm{deg}$. The maneuvering constraints are rudder angle $\delta \in\left[-35^{\circ}, 35^{\circ}\right]$, and propeller revolution $n p \in\left[0, n p_{\max }\right]$. TSs are placed around OS to simulate various encountering situations.

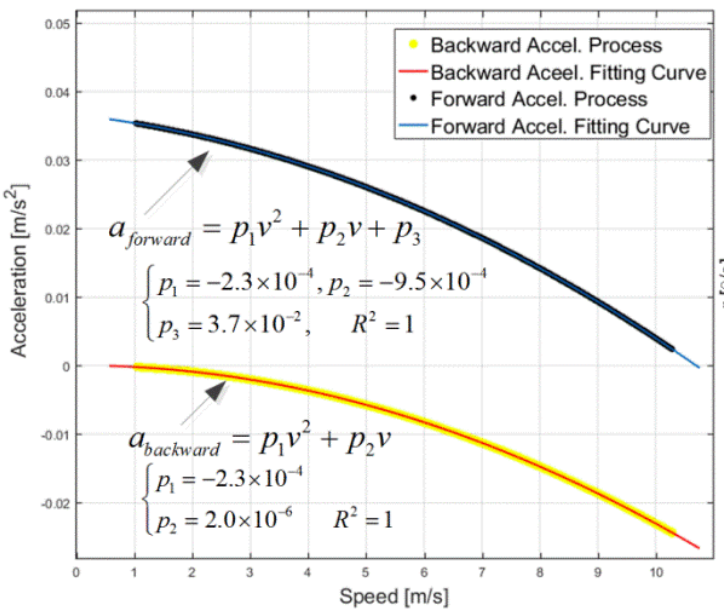

(1)

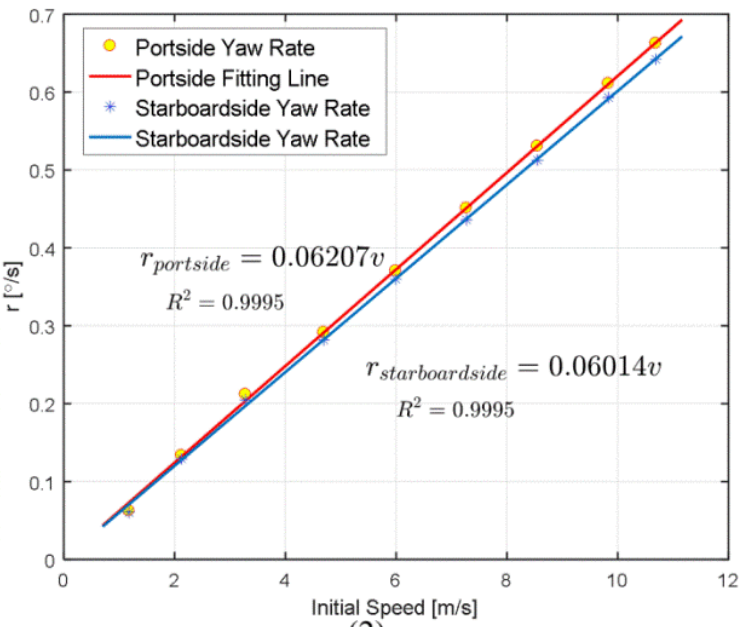

(2)

Fig. 8. The acceleration as a function of speed (1) and the yaw rate as a function of speed (2) (note: the data in the figures are obtained based on the simulations of KVLCC2)

In the simulation, the motion of the OS follows Equation (8) and (9) and time for steering is calculated by Equation (14) - (18). The coefficients $p_{1}, p_{2}, p_{3}$, and $k$ are identified with simulations of KVLCC2. The simulation of KVLCC2 is based on a 3 DOF MMG (Maneuvering Model Group) model. The details of KVLCC2 can be found in the literature (Yasukawa \& Yoshimura, 2015). To identify the coefficients, the acceleration and turning of KVLCC2 are simulated.

In the acceleration simulation, we set the maximal revolution of the propeller as 2.4 revolutions per second (rps). The response of acceleration to velocity is shown in Fig. 8(1). By regression analysis, $p_{1}=-2.312 \times 10^{-4}, p_{2}=-9.484 \times 10^{-4}$ and $p_{3}=3.654 \times 10^{-2}$ are accepted. In deceleration phase, we set the revolution to $0 \mathrm{rps}$, and then $p_{1}=-2.313 \times 10^{-4}, p_{2}=1.987 \times 10^{-6}$ and $p_{3}=0$.

In the simulation of turning, the maximal rudder angle $\left(35^{\circ}\right)$ is applied. The yaw rate responding to velocity is recorded in Fig. 8(2). The performance of port-side turning and starboard-side turning are slightly 
different. The coefficient $\mathrm{k}$ for port-side turning, $k=6.207 \times 10^{-2}$; and for starboard-side turning, $k=6.014 \times 10^{-2}$.

\subsection{Basic Scenarios}

The settings of Basic Scenarios are shown in table I and the layouts are shown in Fig. 9. Four scenarios are involved:

- Scenario 1-1 is a heading situation where a TS is approaching the OS from the OS's stem.

- Scenario 1-2 is a crossing situation in which a TS is placed in the OS's port-side.

- Scenario 1-3 is a crossing situation in which a TS is placed in the OS's starboard-side.

- Scenario 1-4 is an overtaking situation that a TS is pursuing the OS from the OS's aft.

Fig. 10(1) displays the TCR levels for Scenario 1-1, 1-2 and 1-3, and Fig. 10(2) shows the TCR for Scenario 1-4. Since the TS is designed to collide with the OS, the risk increases as time goes on, and equals to 1 at the end. Moreover, the risk levels reach 1 before the collision happens in some scenarios (see Fig. 10 (1)). That means, although a collision has not happened, no maneuver can be taken by the OS to avoid the collision (see Fig. 11 (3)). In the overtaking scenario (Fig. 10 (2)), in the most time, the OS has more than a half chance to avoid the collision. Interestingly, if the OS does not take any actions before TCR level reaches 0.5 , the TCR level will increase dramatically and lead to a collision directly.

Table I. The detailed settings for Basic Scenarios

\begin{tabular}{cccccc}
\hline & OS & \multicolumn{4}{c}{ TS } \\
\cline { 3 - 6 } & & Scenario 1-1 & Scenario 1-2 & Scenario 1-3 & Scenario 1-4 \\
\hline Length[m] & 350 & 150 & 150 & 150 & 150 \\
Position [NM] & $(0,0)$ & $(-0.006,4.818)$ & $(-1,4.092)$ & $(3.349,2.021)$ & $(0.134,-3.998)$ \\
Speed [knots] & 10 & 17 & 18 & 19 & 18 \\
Course [deg] & 0 & 179.9 & 158.8 & 226.1 & 0 \\
\hline
\end{tabular}




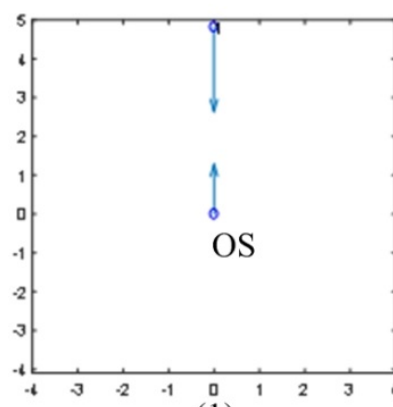

(1)

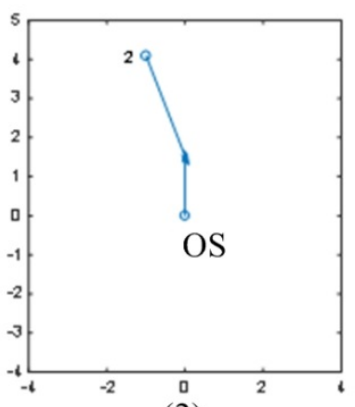

(2)

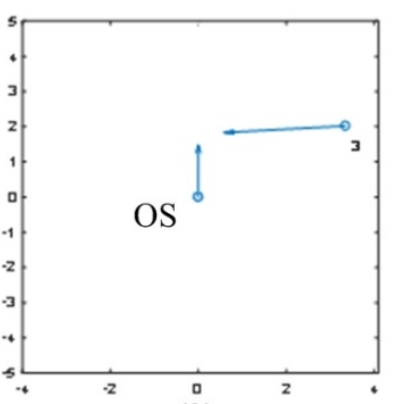

(3)

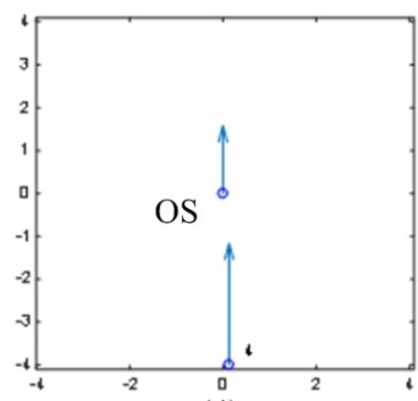

(4)

Fig. 9. The position and directions of the ships in different Scenarios1-1 to Scenario 1-4

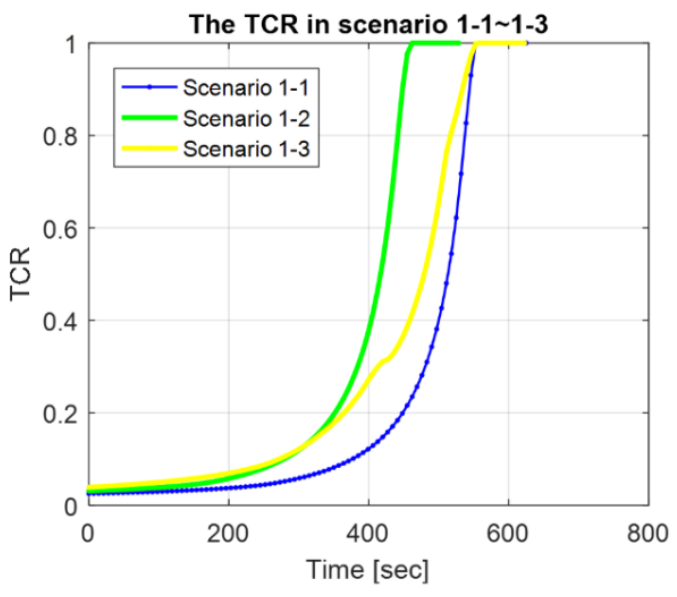

(1)

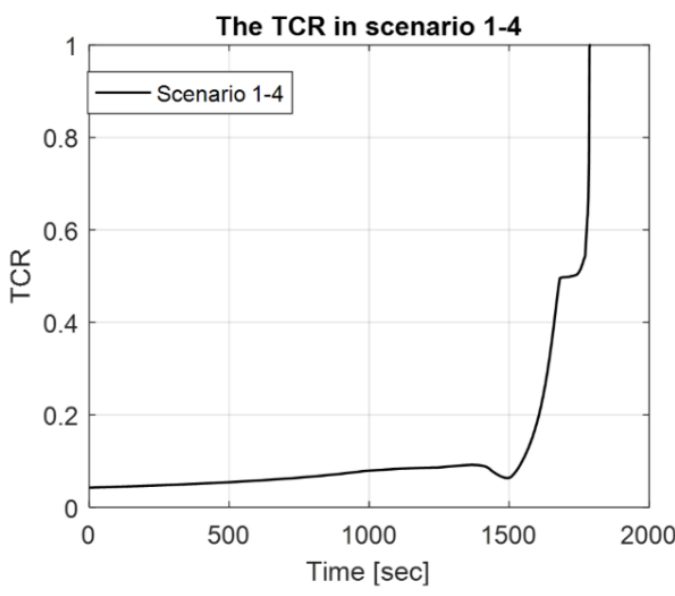

(2)

Fig. 10. The TCR levels in different scenarios

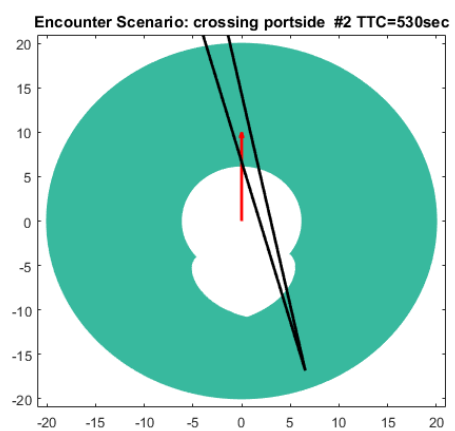

(1)

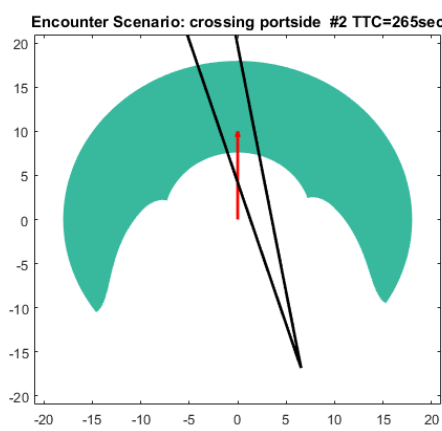

(2)

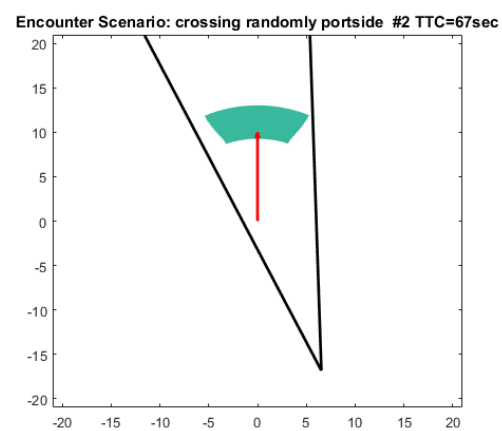

(3)

Fig. 11. The OS's velocity space in Scenario 1-2 at different time slices (the black lines indicate the VO sets at 530, 265 and $67 \mathrm{sec}$. respectively, and the green areas are the RV sets at corresponding time slices.) 
Fig. 11 shows the details about risk calculation of Scenario 1-2 in the OS's velocity space in different time slices. The figures of other scenarios are shown in Appendix A. Fig. 11 (1) shows the VO set and the RV set for $\tau=530 \mathrm{sec}$. At this time, the OS has many alternative velocities that can successfully avoid the collision, thus the risk is relatively low. As time goes on, two ships are approaching each other. Corresponding, the VO set is enlarged and the RV set is shrunk, shown in Fig. 11 (2). When $\tau=67 \sec$ (in Fig. 11 (3)), although the collision has not happened, all the velocity options for the OS are in VO set, which means there is no solution for the OS (collision is unavoidable).

\subsection{Critical Scenarios}

The basic scenarios show the measurement's performance in different types of encounter situations. In this section, we focus on testing the performance of TCR measurement in some similar encounter situations, but different critical levels.

We select the crossing situation of two ships in the scenario design. In these scenarios, the TSs have same velocities, while the positions of TSs are slightly different. The positions are placed to make sure that the TCPA of these TSs is the same, but the DCPAs are different. A bigger DCPA implies the TS will pass OS further away. These scenarios are designed to test whether the proposed model can meet the general knowledge about the risk: a TS passing by the OS nearby has a higher risk level than the one passing by far away. We set the DCPAs ranging from 0 to $0.9 \mathrm{NM}$ in these scenarios. The settings are shown in Table II. The layouts of these scenarios are plotted together in Fig. 12 (1).

Table II. Details of the Critical Scenarios

\begin{tabular}{ccccccc}
\hline & OS & Scenario 2-1 & Scenario 2-2 & Scenario 2-3 & Scenario 2-4 & Scenario 2-5 \\
\cline { 3 - 7 } & & TS 1 & TS 2 & TS3 & TS 4 & TS 5 \\
\hline Position & $(0,0)$ & $(3.378,3.451)$ & $(3.270,3.556)$ & $(3.163,3.661)$ & $(2.949,3.870)$ & $(2.735,4.080)$ \\
[NM] & & 0 & 0.15 & 0.3 & 0.6 & 0.9 \\
DCPA [NM] & & 150 & 150 & 150 & 150 & 150 \\
Length [m] & 350 & 16.4 & 16.4 & 16.4 & 16.4 & 16.4 \\
Speed [knots] & 10 & 249.6 & 249.6 & 249.6 & 249.6 & 249.6 \\
Course [deg] & 0 & & & & & \\
\hline
\end{tabular}




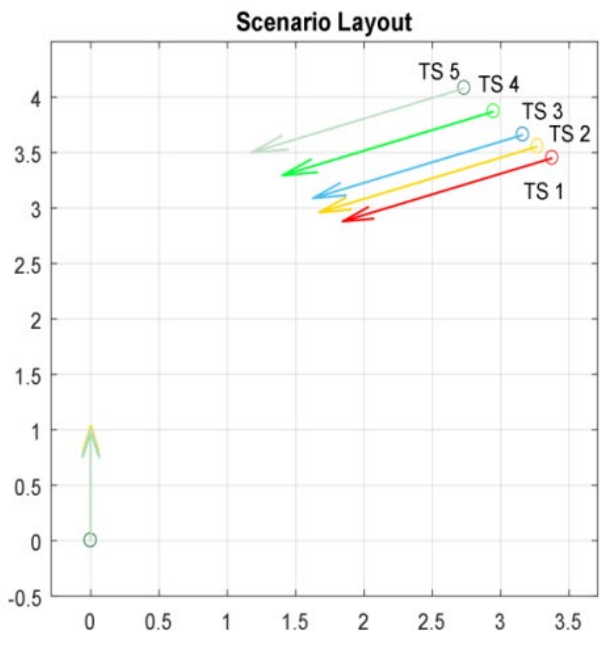

(1)

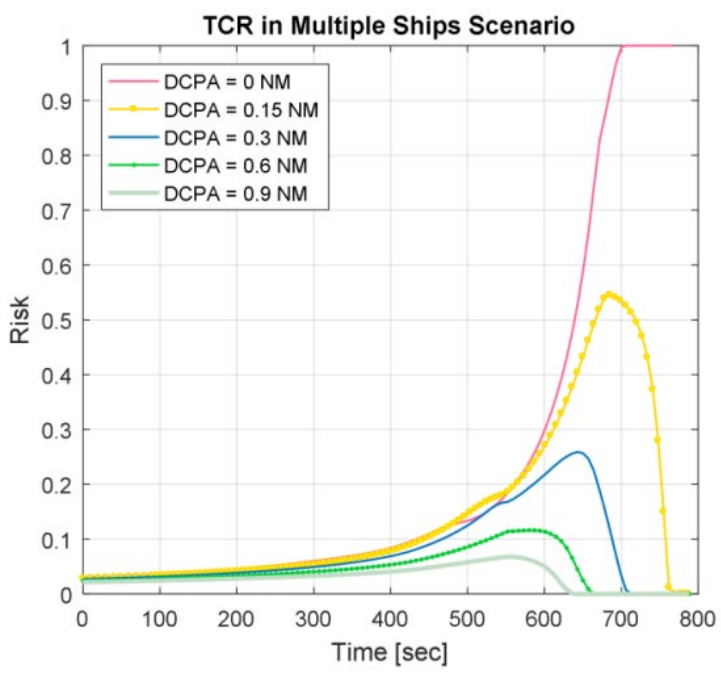

(2)

Fig. 12. The positions and directions of the ships and the TCR levels in Scenario 2-1 to Scenario 2-5

Fig. 12 (2) shows the TCR levels for these 5 crossing scenarios. The most dangerous scenario is the TS1 scenario, in which TS1 approaches to the OS and passes it without any additional distance (DCPA = 0). At the end, TS1 collides with the OS and the risk rises to 1 . The second dangerous scenario is TS2 scenario where TS2 passes the OS at $0.15 \mathrm{NM}$. Considering the dimensions of the ship, this distance is dangerous. Since collision not happens, the TCR drops after it reaches the peak over 0.5 (the orange curve in the figure). The rest of the curves have a similar property, their TCRs have a negative relation with DCPA. In fact, we can observe that a larger DCPA implies a lower TCR level, which is in line with common sense.

\subsection{Mixed Scenarios}

Multiple ships are introduced to test the performance of the TCR measurement under a complex situation. We are interested in how the TCR level changes over time when one more ship is added. Two scenarios are employed. In each scenario, three ships are involved. In Scenario 3-1, one TS is set to collide with OS, while the other can pass OS safely (DCPA is huge); in Scenario 3-2, two TSs are designed to collide with the OS. The details of settings are shown in Table III.

The TCR level in Scenario 3-1 is shown in Fig. 13 (2). The blue line shows the total TCR when two ships appear together; the red line represents the TCR level when TS6 occurs alone; the cyan line for the situation when TS7 appears alone. Since the OS inevitably collides with TS6, the total TCR level goes to 1 
at the end. Comparing the bold blue curve with the red line, introducing one ship will increase the TCR level at each time slice, though the introducing ship (TS3) would not collide with the OS. This finding is in line with common sense: introducing more objects in one area increases the collision dangers. In fact, even the added ship (say TS 7) would not lead to a collision, it could block some collision-free solutions for avoiding collision with the original target ship (say TS6), as shown in Fig. 13(3).

The increase of the total TCR level depends on the relations of VO sets in the OS's velocity space. In the Scenario 3-1, since these VO sets do not overlap each other (Fig. 13(3)), the total TCR level is the sum of the TCR levels from TSs when they appear alone. However, in the Scenario 3-2 (Fig. 14), the VO sets from TSs are overlapping all the time. The TCR level is smaller than the sum of TCRs from a single ship (the dash-line in Fig. 14(2)) and larger than any TCR from a single ship (the red and cyan lines in Fig. 14(2)).

Table III. The details of Multiple ship scenarios

\begin{tabular}{cccc|cc}
\hline & OS & TS 6 & TS7 & TS 8 & TS 9 \\
\hline Position [NM] & $(0,0)$ & $(3.378,3.451)$ & $(2.949,3.870)$ & $(3.463,3.367)$ & $(3.292,3.535)$ \\
DCPA [NM] & & 0 & 0.6 & 0 & 0 \\
Length [m] & 350 & 150 & 150 & 150 & 150 \\
Speed [knots] & 10 & 16.4 & 16.4 & 16.4 & 16.4 \\
Course [deg] & 0 & 249.6 & 249.6 & 249.6 & 249.6 \\
\hline
\end{tabular}

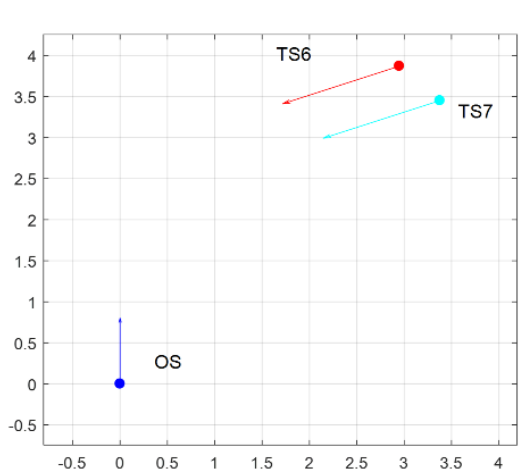

(1)

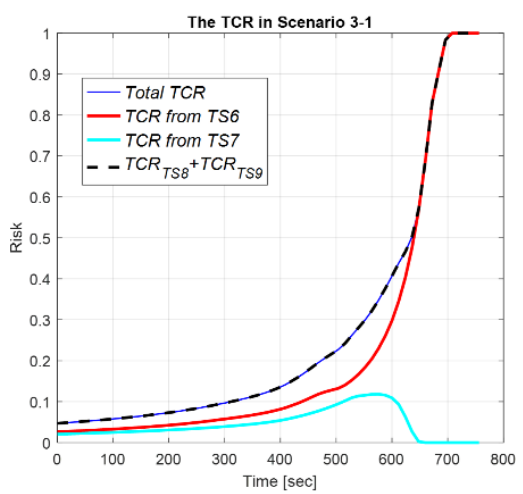

(2)

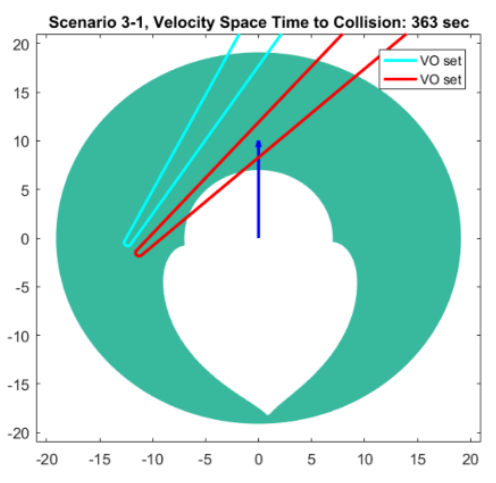

(3)

Fig. 13. The layout, the TCR levels and velocity space (at $363 \mathrm{sec}$.) in Scenario 3-1 


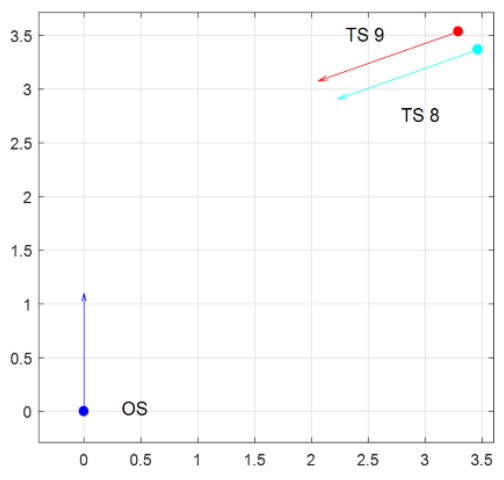

(1)

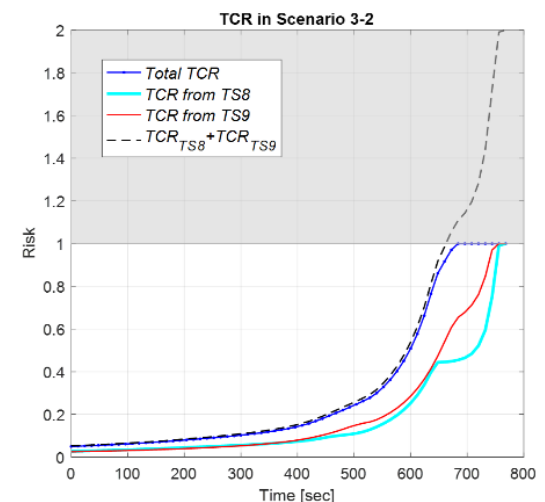

(2)

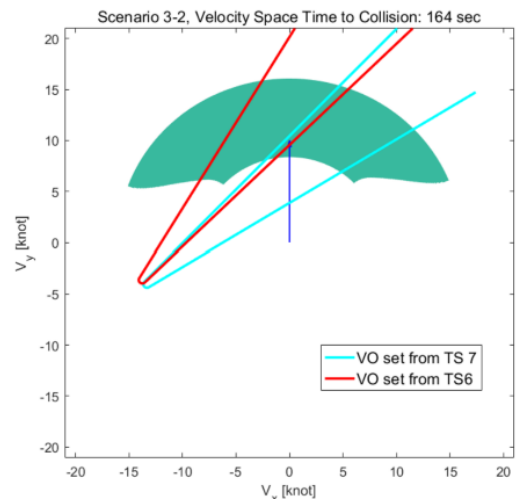

(3)

Fig. 14. The layout, the TCR levels and velocity space (at $363 \mathrm{sec}$.) in Scenario 3-2

\subsection{Regulatory Scenario}

This scenario is designed to see the change of TCR when the regulations are applied, specifically, ships will comply with the International Regulations for Preventing Collisions at Sea 1972 (COLREGs 72)(IMO, 1972). The same crossing situation in Scenario 2-1 is employed, while the ships will follow the regulations.

According to COLREGs 72, the OS in this situation is the "give-way vessel", the TS is the "stand-on vessel" and two ships should avoid turning to port. Hence, the portside maneuvers, which against the regulations, needs to be removed from the OS's RV set. We remove those maneuvers and obtain a new RV set. Correspondingly, the calculation of TCR is updated. The left panel in Fig. 15 shows the new RV set at TTC $=364 \mathrm{sec}$. where the portside turnings are excluded; the right panel shows the updated TCR level overtime (the red curve) and original TCR level (grey curve).

In this scenario, since portside maneuvers are banned, some reachable velocities against the regulations are excluded in RV set. In this scenario, the most of these banned maneuvers are collision-free, see Fig. 15(1). Thus, the collision risk, in this case, would be slightly higher than the original one (Fig. 15(2)). This result implies that the OS needs to take a COLREGs-compliant maneuver earlier when it wants to bear the same risk level. 


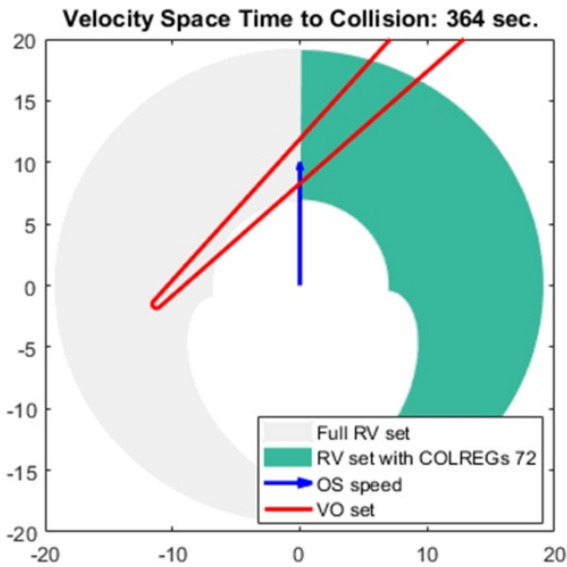

(1)

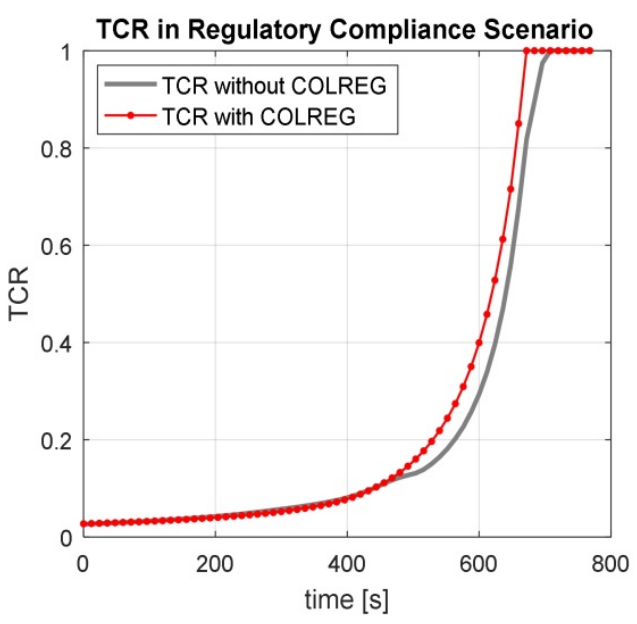

(2)

Fig. 15. The velocity space (at 364 sec.) and TCR levels in Regulatory Scenario

\section{DISCUSSION}

The simulation experiments in Section 5 show that the proposed TCR measurement can reflect the risk level over time and meet our general knowledge. The TCR level increases over time in the Scenarios where two ships are placed to collide. The TCR level increases and then decreases when two ships approach each other and pass safely at the end. In multiple-ship scenarios, the TCR level is higher than the TCR level in two-ship scenarios, even if the extra ships would not lead to a collision. This measurement can support OOWs to foresee the collision risk at present and in the future. Moreover, the proposed measurement is helpful to find a collision-free velocity for the own ship when ships are compliant with regulations.

For simplification purpose, we introduce some assumptions and reduce the uncertainties in the proposed TCR measurement. However, it does not mean this theoretical measurement cannot be implemented in practice. Actually, the proposed TCR measurement is capable to include these uncertainties.

(1) Uncertainty in the construction of VO set

According to Assumption (2), we presume the trajectory of target ship is known and linear. Hence, the constructed VO set is deterministic. That means, $P\left(\right.$ collision $\left.\mid \vec{v}_{i}\right)$ is deterministic, which is either 0 or 1.

In practice, the motions of target ship are usually non-linear and unknown. By prediction algorithms, we only can predict the trajectory with a certain probability. In this case, VO set is not deterministic but 
probabilistic. A velocity inside a VO set will result in a collision with a specified probability(Fulgenzi, Spalanzani, \& Laugier, 2007; Huang, van Gelder, \& Wen, 2018). That means, $P\left(\right.$ collision $\left.\mid \vec{v}_{i}\right)$ falls in a range $[0,1]$. Hence, the calculated TCR can show the expected chance that the own ship cannot avoid a collision with the ships whose trajectories are uncertain.

(2) Uncertainties in the construction of RV set

In Assumption (4)-(5), the environmental disturbance is neglected and the simplified motion model can describe the motion of the ship. That means, the constructed RV set is deterministic. Thus, a velocity in the $\mathrm{RV}$ set is always feasible before the collision, and this argument is guaranteed.

However, ships usually sail with complicated conditions which include wind, wave, etc. These factors strongly affect the maneuverability of ship and the RV set. For example, some reachable speeds in calm water could be unfeasible in a strong current condition; the yaw rates of port turning and starboard turning would be dramatically different. As a consequence, a velocity in the RV set is feasible with a specified probability. Correspondingly, the calculated TCR would show the expected value of the chance that the OS cannot avoid the collision under certain stochastic disturbances.

\section{CONCLUSION}

Collision risk measurement is a vital step for supporting situational awareness at sea. In this paper, we proposed a collision risk measurement considering the capability of a ship to prevent collisions. In particular, the ship maneuverability is taken into account and the percentage of reachable maneuvers leading to collision is formulated as Time-varying Collision Risk (TCR). The value of the TCR directly reflects the Officer On Watch (OOW)'s room for maneuver to avoid a collision, which helps they be aware of the safety level of approaching ships. This method considers both the danger level of approaching "hazards" (the target ships) and the performance of "barriers" (the maneuverability of the own ship), which provides a new perspective on collision risk assessment for mariners and researchers.

In the proposed method, the TCR is indicated by Velocity Obstacle (VO) set and Reachable Velocity (RV) set: VO set, which collects the velocities leading to collisions, is constructed by velocity obstacle 
algorithm; RV set, which gathers the reachable velocities, is constructed by a numerical reachability analysis. The complement of the VO set in the RV set is the set of potential collision-free maneuvers, which can facilitate the OOWs to prevent collisions.

Simulation experiments are carried out to show the potential of the TCR measurement under different scenarios. The results show the proposed method is capable to capture the changes of risk over time, and it helps to provide potential collision-free solutions to the OOWs. Moreover, we simulate a crossing scenario to show the capability of the proposed method in compliance with regulations (COLREGs 72). Multiple ships encounter situations are also simulated to show that introducing an additional ship to the original scenario will increase the risk of collision, even if the introducing ship would not collide with the own ship.

The proposed TCR measurement is an initial step for evaluating the collision risk for real-time collision prevention. Future research should focus on the following aspects. Firstly, different distributions of maneuver choice will be applied with the TCR measurement to cover the uncertainties in maneuvers. Secondly, Nonlinear VO algorithm and Probabilistic VO algorithm will be considered to handle the uncertainty of the target ship's motion(Huang et al., 2018). Thirdly, the impacts of environmental disturbances will also be considered with a more reasonable ship motion model. Finally, more encounter scenarios in compliance with regulations will be introduced to support safer evasive actions.

\section{ACKNOWLEDGEMENT}

This research was funded by the China Scholarship Council under Grant: 201406950010. We thank M.B. Mendel for his comments on earlier versions of the manuscript and Linying Chen for her assistance in revising the manuscript. We also would like to thank anonymous reviewers for comments that greatly improved the manuscript.

\section{Appendix A}

In this appendix, the velocity space of OS in basic scenarios is shown. The setting of these scenarios can be found in Section 5.2. 

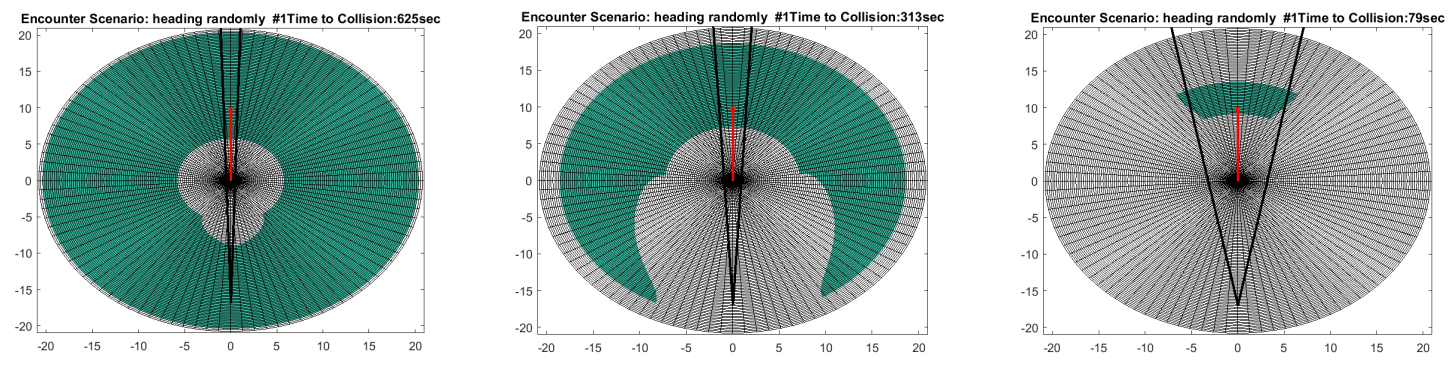

Figure A1. The OS's velocity space in Scenario 1-1 at time to collision: 530, 313 and 79 sec.

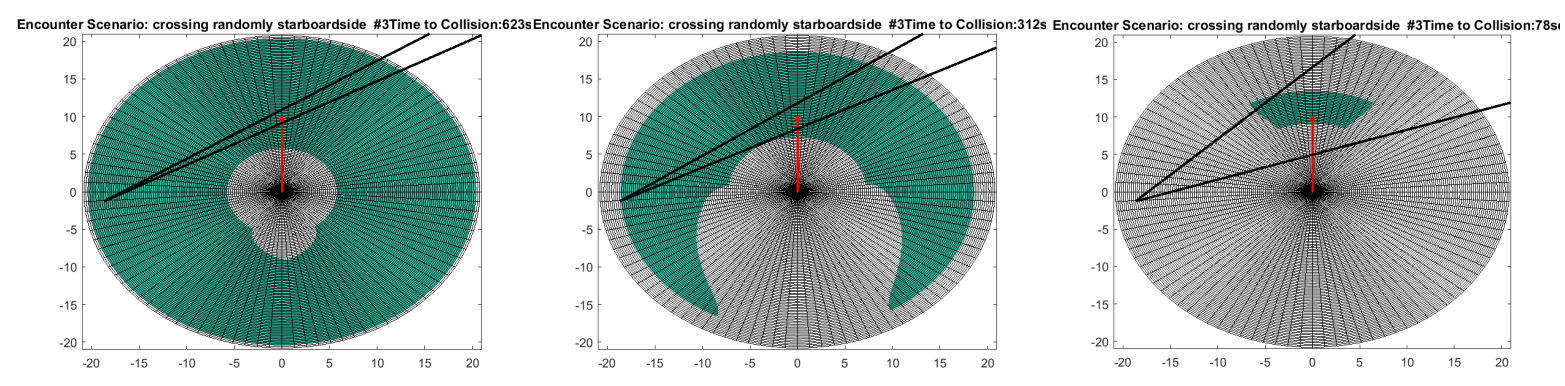

Figure A2. The OS's velocity space in Scenario 1-3 at TTC: 623, 312 and $78 \mathrm{sec}$.
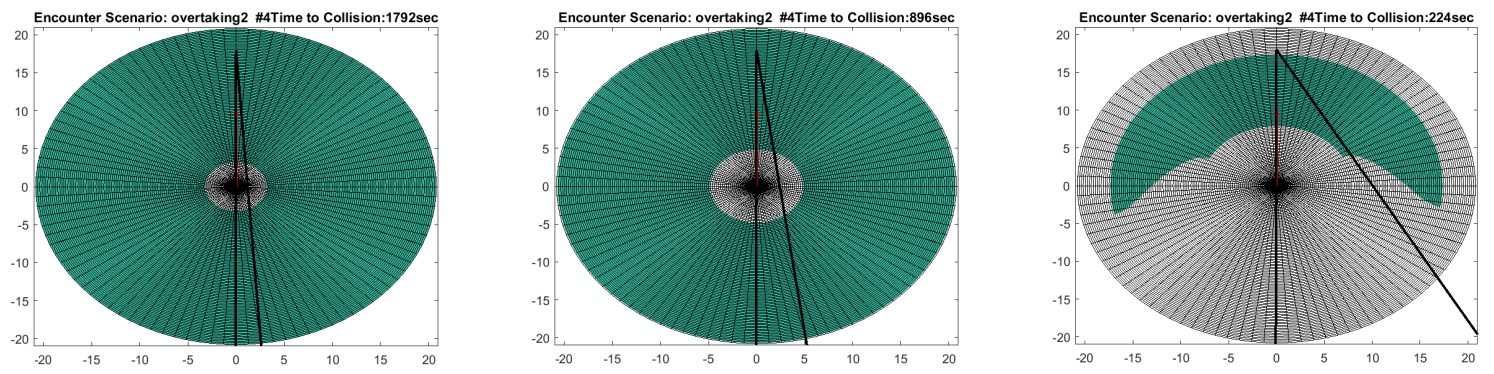

Figure A3. The OS's velocity space in Scenario 1-4 at TTC: 1792, 896 and $224 \mathrm{sec}$.

\section{REFERENCE}

Ahn, J.-H., Rhee, K.-P., \& You, Y.-J. (2012). A study on the collision avoidance of a ship using neural networks and fuzzy logic. Applied Ocean Research, 37, 162-173. doi:10.1016/j.apor.2012.05.008

Althof, M. (2010). Reachability Analysis and its Application to the Safety Assessment of Autonomous Cars. $(\mathrm{PhD})$, Technische Universität München, Germany.

Andretta, M. (2014). Some considerations on the definition of risk based on concepts of systems theory and probability. Risk Anal, 34(7), 1184-1195. doi:10.1111/risa.12092

Aven, T. (2012). The risk concept - historical and recent development trends. Reliability Engineering \& System Safety, 99, 33-44. doi:10.1016/j.ress.2011.11.006 
Baldauf, M., Benedict, K., Fischer, S., Motz, F., \& Schröder-Hinrichs, J. U. (2011). Collision avoidance systems in air and maritime traffic. Proceedings of the Institution of Mechanical Engineers, Part O: Journal of Risk and Reliability, 225(3), 333-343. doi:10.1177/1748006x11408973

Belkhouche, F., \& Bendjilali, B. (2012). Dynamic collision risk modeling under uncertainty. Robotica, 31(04), 525-537. doi:10.1017/s0263574712000550

Chauvin, C., Lardjane, S., Morel, G., Clostermann, J. P., \& Langard, B. (2013). Human and organisational factors in maritime accidents: analysis of collisions at sea using the HFACS. Accid Anal Prev, 59, 2637. doi:10.1016/j.aap.2013.05.006

Chin, H. C., \& Debnath, A. K. (2009). Modeling perceived collision risk in port water navigation. Safety Science, 47(10), 1410-1416. doi:10.1016/j.ssci.2009.04.004

Crane, C. L., Eda, H., \& Landsburg, A. (1989). Controllability. In E. V. Lewis (Ed.), Principles of Naval Architecture (Vol. 3). Jersey, US: The SNAME.

Fiorini, P., \& Shiller, Z. (1998). Motion Planning in Dynamic Environments using Velocity Obstacle. The International Journal of Robotics Research, 17(7), 760-772.

Fulgenzi, C., Spalanzani, A., \& Laugier, C. (2007). Combining Probabilistic Velocity Obstacles and Occupancy Grid for safe Navigation in dynamic environments. Paper presented at the Workshop on Safe Navigation, Rome, Italy. 2007.

Goerlandt, F., \& Montewka, J. (2015). Maritime transportation risk analysis: Review and analysis in light of some foundational issues. Reliability Engineering \& System Safety, 138, 115-134. doi:10.1016/j.ress.2015.01.025

Goerlandt, F., Montewka, J., Kuzmin, V., \& Kujala, P. (2015). A risk-informed ship collision alert system: Framework and application. Safety Science, 77, 182-204. doi:10.1016/j.ssci.2015.03.015

Grech, M. R., Horberry, T., \& Smith, A. (2002, Sept 30 - Oct 4). Human error in maritime operations: Analyses of accident reports using the Leximancer tool Paper presented at the Proceedings of the Human Factors and Ergonomics Society Annual Meeting, Baltimore, U.S.

Hermansson, H. (2012). Defending the conception of "objective risk". Risk Anal, 32(1), 16-24. doi:10.1111/j.1539-6924.2011.01682.x

Hilgert, H., \& Baldauf, M. (1997). A Common Risk Model for the Assessment of Encounter Situations on Board Ships German Journal of Hydrography, 49(4), 531-542.

Huang, Y., Chen, L., \& van Gelder, P. H. A. J. M. (2019). Generalized velocity obstacle algorithm for preventing ship collisions at sea. Ocean Engineering, 173, 142-156. doi:10.1016/j.oceaneng.2018.12.053

Huang, Y., \& Gelder, P. H. A. J. M. V. (2017). Non-linear velocity obstacles with applications to the maritime domain. Paper presented at the the 17th International Congress on Maritime Transportation and Harvesting of Sea Resources, Lisbon, Portugal.

Huang, Y., van Gelder, P. H. A. J. M., \& Wen, Y. (2018). Velocity Obstacle Algorithms for Collision Prevention at Sea. Ocean Engineering, 151,308-321.

Convention on the International Regulations for Preventing Collisions at Sea, 1972 (COLREGs), (1972).

Kao, S.-L., Lee, K.-T., Chang, K.-Y., \& Ko, M.-D. (2006). A Fuzzy Logic Method for Collision Avoidance in Vessel Traffic Service. Journal of Navigation, 60(01), 17-31. doi:10.1017/s0373463307003980

Kuwata, Y., Wolf, M. T., Zarzhitsky, D., \& Huntsberger, T. L. (2014). Safe maritime autonomous navigation with COLREGS, using velocity obstacles. IEEE Journal of Oceanic Engineering, 39(1), 110119. doi:10.1109/JOE.2013.2254214

Lambert, A., Gruyer, D., \& Saint Pierre, G. (2008). A Fast Monte Carlo Algorithm for Collision Probability Estimation. 10th International Conference on Control Automation Robotics \& Vision: ICARV 2008, Vols 1-4, 406-411. doi:Doi 10.1109/Icarcv.2008.4795553

Li, S., Meng, Q., \& Qu, X. (2012). An overview of maritime waterway quantitative risk assessment models. Risk Anal, 32(3), 496-512. doi:10.1111/j.1539-6924.2011.01697.x

Li, Y., Landsburg, A. C., Barr, R. A., \& Cahsal, S. M. (2005). Improving ship maneuverability standards as a means for increasing ship controllability and safety. Oceans 2005, Vols 1-3, 1972-1981. 
Liu, J., Hekkenberg, R., Rotteveel, E., \& Hopman, H. (2015). Literature review on evaluation and prediction methods of inland vessel manoeuvrability. Ocean Engineering, 106, 458-471. doi:10.1016/j.oceaneng.2015.07.021

Liu, Y., Liu, W., Song, R., \& Bucknall, R. (2017). Predictive navigation of unmanned surface vehicles in a dynamic maritime environment when using the fast marching method. International Journal of Adaptive Control and Signal Processing, 31(4), 464-488. doi:10.1002/acs.2561

Lopez-Santander, A., \& Lawry, J. (2016). An Ordinal Model of Risk Based on Mariner's Judgement. Journal of Navigation, 70(02), 309-324. doi:10.1017/s0373463316000576

Martins, M. R., \& Maturana, M. C. (2010). Human error contribution in collision and grounding of oil tankers. Risk Anal, 30(4), 674-698. doi:10.1111/j.1539-6924.2010.01392.x

Montewka, J., Ehlers, S., Goerlandt, F., Hinz, T., Tabri, K., \& Kujala, P. (2014). A framework for risk assessment for maritime transportation systems - A case study for open sea collisions involving RoPax vessels. Reliability Engineering \& System Safety, 124, 142-157. doi:10.1016/j.ress.2013.11.014

Park, J., \& Kim, J. (2016). Predictive Evaluation of Ship Collision Risk Using the Concept of Probability Flow. IEEE Journal of Oceanic Engineering(99), 1-10.

Sahawneh, L. R., \& Beard, R. W. (2014). A Probabilistic Framework for Unmanned Aircraft Systems Collision Detection and Risk Estimation. 2014 Ieee 53rd Annual Conference on Decision and Control (Cdc), 242-247.

Simsir, U., Amasyali, M. F., Bal, M., Çelebi, U. B., \& Ertugrul, S. (2014). Decision support system for collision avoidance of vessels. Applied Soft Computing Journal, 25, 369-378. doi:10.1016/j.asoc.2014.08.067

Szlapczynski, R., \& Szlapczynska, J. (2016). An analysis of domain-based ship collision risk parameters. Ocean Engineering, 126, 47-56. doi:10.1016/j.oceaneng.2016.08.030

Tam, C., \& Bucknall, R. (2010). Collision risk assessment for ships. Journal of marine science and technology, 15(3), 257-270. doi:10.1007/s00773-010-0089-7

Tam, C., Bucknall, R., \& Greig, A. (2009). Review of Collision Avoidance and Path Planning Methods for Ships in Close Range Encounters. Journal of Navigation, 62(03), 455-476. doi:10.1017/s0373463308005134

van den Berg, J., Lin, M., \& Manocha, D. (2008). Reciprocal Velocity Obstacles for real-time multi-agent navigation. Paper presented at the 2008 IEEE International Conference on Robotics and Automation ( ICRA), Pasadena, CA, USA. <Go to ISI $>$ ://WOS:000258095001101

Velasco, G. A. M., Borst, C., Ellerbroek, J., van Paassen, M. M., \& Mulder, M. (2015). The Use of Intent Information in Conflict Detection and Resolution Models Based on Dynamic Velocity Obstacles. Ieee Transactions on Intelligent Transportation Systems, 16(4), 2297-2302. doi:10.1109/Tits.2014.2376031

Vendittelli, M., Laumond, J.-P., \& Nissoux, C. (1999). Obstacle Distance for Car-Like Robots. IEEE Transactions on Robotics and Automation, 15(4), 678-691.

Wang, N. (2010). An Intelligent Spatial Collision Risk Based on the Quaternion Ship Domain. Journal of Navigation, 63(04), 733-749. doi:10.1017/s0373463310000202

Yasukawa, H., \& Yoshimura, Y. (2015). Introduction of MMG standard method for ship maneuvering predictions. Journal of marine science and technology, 20(1), 37-52. doi:DOI 10.1007/s00773-0140293-y

Zhang, J., Teixeira, A. P., Guedes Soares, C., Yan, X., \& Liu, K. (2016). Maritime Transportation Risk Assessment of Tianjin Port with Bayesian Belief Networks. Risk Anal, 36(6), 1171-1187. doi:10.1111/risa.12519

Zhang, W., Goerlandt, F., Montewka, J., \& Kujala, P. (2015). A method for detecting possible near miss ship collisions from AIS data. Ocean Engineering, 107, 60-69. doi:10.1016/j.oceaneng.2015.07.046 